\title{
Nonseparable Multidimensional Perfect Reconstruction Filter Banks and Wavelet Bases for $\mathscr{R}^{n}$
}

\author{
Jelena Kovačević, Member, IEEE, and Martin Vetterli, Senior Member, IEEE
}

\begin{abstract}
Although filter banks have been in use for more than a decade, only recently have some results emerged, setting up the theory of general, nonseparable multidimensional filter banks. At the same time, wavelet theory emerged as a useful tool in many different fields of pure and applied mathematics as well as in signal analysis. Recently, it has been shown that the two theories are closely related. Not only does the filter bank perform a discrete wavelet transform, but also under certain conditions it can be used to construct continuous bases of compactly supported wavelets. For multidimensional filter banks, using arbitrary sampling lattices, conditions for perfect reconstruction are given. The orthogonal case is analyzed indicating orthogonality relations between the filters in the bank and their shifts on the sampling lattice. A linear phase condition follows, as a tool for testing or building banks containing linear phase (symmetric) filters. It is shown how, in some cases, nonseparable filters can be implemented in a separable fashion. The two-channel case in multiple dimensions is studied in detail: the form of a general orthogonal solution is given and possible linear phase solutions are presented, showing that orthogonality and symmetry are exclusive, independent of the number of dimensions (assuming real FIR filters). Attractive cascade structures with specific properties (orthogonality and linear phase) are proposed. For the four-channel two-dimensional case, filters being orthogonal and symmetric are obtained, a solution that is impossible using separable filters. We also discuss methods for obtaining multidimensional filters from their one-dimensional counterparts. Next, we make a connection to nonseparable wavelets through the construction of iterated filter banks. As suming the $L^{2}$ convergence of the scaling function, we show that as in the one-dimensional case, the scaling function satisfies a two-scale equation, and the wavelets are orthogonal to each other and their scales and translates (as well as to the scaling function). Then, for the scaling function to exist, we show that it is necessary that the low-pass filter have a zero at aliasing frequencies. Following the discussion on the choice of the dilation matrix, an interesting "dragon" is constructed for the hexagonal case. For the two-channel case in multiple dimensions it is shown that the wavelets defined previously indeed constitute a basis for $L^{2}\left(\mathscr{R}^{n}\right)$ functions. Following the result on necessity of a zero, we conjecture that the low-pass filter can be made regular by putting a zero of sufficiently high order at aliasing frequencies. Based on this, a small orthonormal low-pass filter is designed for which we conjecture that it would lead to a continuous scaling function, and thus, wavelet basis. $A$ biorthogonal example is also given.
\end{abstract}

Manuscript received February 18, 1991; revised July 15, 1991. This work was supported by the National Science Foundation under Grants ECD-88 11111 and MIP-90-14189.

J. Kovačević is with AT\&T Bell Laboratories, Murray Hill, NJ 07974

M. Vetterli is with the Department of Electrical Engineering and the Center for Telecommunications Research, Columbia University, New York, NY 10027

IEEE Log Number 9105035.
Index Terms-Multidimensional, nonseparable filter banks, wavelets, multidimensional wavelets, filter banks.

\section{INTRODUCTION}

QINCE the introduction of digital multirate filter banks for the compression of speech signals 15 years ago [8], they have been widely used mainly for subband coding of speech, still images, and video [2], [37], [43], [47]. The underlying theory progressed from cancellation of aliasing (or repeated spectra), to building systems achieving exact reconstruction of the signal, and from two-channel orthogonal banks [26], [30], to general multichannel systems [31], [33], [38], [39], [44]. For implementational reasons all of these efforts concentrated on filters having rational transfer functions.

Independent of this work, the theory of wavelets was developed in applied mathematics [9], [17], [23], [25]. With the work of Daubechies [9], Mallat [23], and Meyer [25], it became clear that filter banks and wavelets were closely related. Filter banks compute the equivalent of a discrete wavelet transform, and under certain conditions (regularity of the low-pass filter), they can be used to derive continuous bases of wavelets [9].

To explain briefly how a subband system works, refer to Fig. 1, where a general multidimensional $N$-channel system is shown. The input signal is fed through $N$ branches, each one containing a bandpass filter, and subsequently subsampled by $N=\operatorname{det} D$ to its new multidimensional Nyquist frequency $(D$ is a sublattice of the input lattice and has a sampling density that is $N$ times smaller). Then each channel signal (subband) is encoded, transmitted, and decoded. To resynthesize the original signal, one has to upsample all the subbands back to the original lattice and pass them through a set of bandpass synthesis filters. Note that the basic blocks in the system perform filtering, sampling rate change, and coding / transmission/decoding. In what follows, we will be concerned only with the former two parts and we will assume that coding/transmission/decoding is performed in a lossless fashion. Another assumption will be that the sampling density and the number of channels are the same so as to preserve the same number of samples through the various steps of the system. This will be referred to as a critically sampled filter bank.

Consider the sampling part of the system. While in one dimension sampling by $N$ can be performed in only one way, 


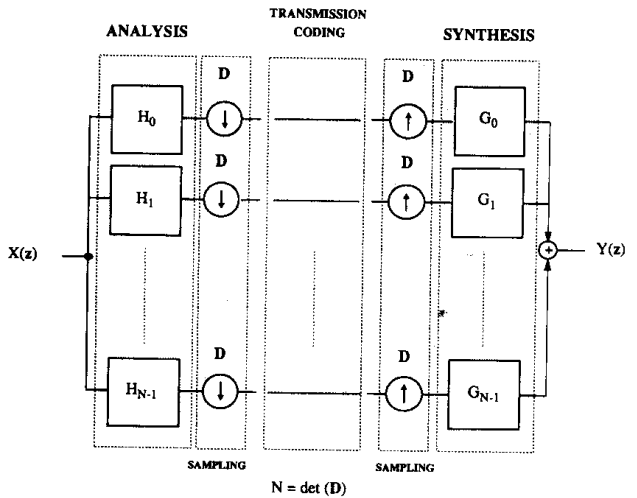

Fig. 1. An analysis/synthesis $n$-dimensional filter bank.

in two or more dimensions this is not true any more. Multidimensional sampling is represented by a lattice which can be separable or nonseparable. In most of the previous work on two- or three-dimensional multirate processing the sampling rate changes which are used are separable and can be performed along one dimension at a time. However, when dealing with multidimensional signals, true multidimensional processing is more appropriate. Recently, some results have emerged where nonseparable sampling is used, mostly for two-dimensional systems (see [2], [20], [21], [22], [37], [45], [46]). In [48], nonseparable filters were used on a hexagonally sampled input signal, which was then separably subsampled, leading to an interesting directional analysis of images. Aliasing was cancelled, and perfect reconstruction was well approximated.

As for the filtering part, there are a number of questions of interest that have to be addressed. They include design constraints, such as orthogonality, linear phase (symmetry), and regularity. At the same time, the filters themselves can be separable or nonseparable regardless of the sampling lattice. Obviously, while separable filters offer the advantage of low-complexity processing, their nonseparable counterparts have more degrees of freedom and hence allow better designs. In multirate filtering, a nonseparable filter can sometimes still be implemented in a separable fashion, and this will be explored as well. In what follows, unless stated otherwise, we will assume real finite impulse response (FIR), or compactly supported, filters.

If we consider the filter bank system as a whole, two issues of interest arise, namely alias-free reconstruction and perfect reconstruction. The former means that, from input to output, the system can be regarded as a shift-invariant filter, while the latter requires the reconstructed signal to be equal to the input signal (possibly within a delay and a scale factor).

Finally, we would like to use perfect reconstruction filter banks in order to derive wavelet bases. The key construction is the iteration of the filter bank along its low-pass branch. If the low-pass filter is regular (its iterated version converges to a well-defined, possibly smooth function), this construction leads to a wavelet basis, as was first shown in the one- dimensional case and subsampling by 2 by Daubechies [9]. This was also studied under the framework of multiresolution analysis in [23], [25]. In one dimension, subsampling by $N$ in an $N$-branch filter bank leads, after iteration, to a scaling function $\phi(x)$ satisfying a two-scale equation of the form

$$
\phi(x)=\sum_{n} c_{n} \phi(N x-n)
$$

as well as to $(N-1)$ wavelets which are also linear combinations of $\phi(N x-n)$ [41]. The most studied case has been for $N=2$.

In multiple dimensions the situation is more complicated. From a discrete filtering point of view, subsampling is defined by a sublattice of the original lattice (which we can assume, without loss of generality, to be $\mathscr{Z}^{n}$ ). The sublattice is represented by a dilation matrix $D$ (the equivalent of the subsampling, or dilation factor, $N$ in the one-dimensional case). The indexes of points belonging to the sublattice are given as weighted integer combinations of the columns of $D$. For example, the following matrices are possible representations of the so-called two-dimensional quincunx sublattice [2], [7], [15], [37], [43]

$$
\begin{array}{ll}
D_{1} & =\left(\begin{array}{rr}
1 & 1 \\
1 & -1
\end{array}\right), \quad D_{2}=\left(\begin{array}{rr}
1 & -1 \\
1 & 1
\end{array}\right), \\
D_{3} & =\left(\begin{array}{ll}
2 & 1 \\
0 & 1
\end{array}\right) .
\end{array}
$$

Its sampling lattice is given in Fig. 2 showing that one out of two points is retained (this is the only nonseparable sublattice with $|\operatorname{det} \boldsymbol{D}|=2$ ). Thus, using this lattice would result in a two-dimensional nonseparable two-channel case.

Now, when iterating the filter bank, we iterate the subsampling by $D$, that is, the overall subsampling corresponds to an integer power of $D$. This can be very different for different matrices $D$ (e.g., $D_{1}^{2}=2 I$ while $D_{3}^{n}$ will never be separable).

The scaling function derived from the iterated filter bank (if it exists) will also obey a two-scale equation (1) which now depends on $D$. One necessary requirement on the matrix $D$ is that it be a dilation in all dimensions (otherwise, an associated wavelet analysis would not increase resolution in all dimensions [7]). This is equivalent to requiring that all eigenvalues of $\boldsymbol{D}$ should have magnitude strictly greater than 1 , and so, for example, $\boldsymbol{D}_{3}$ is not a valid dilation matrix (it dilates by 2 in the $[x, 0]$ direction, but does not dilate at all in the $[x,-x]$ direction).

The vastly different behavior of iterated filter banks depending on the matrix $\boldsymbol{D}$ was most strikingly demonstrated by Gröchenig and Madych [16], as well as Lawton and Resnikoff [49]. In [16], the authors showed iterations of very simple filters (essentially Haar filters) that produce fractal, self-similar tilings of the space for certain matrices, while giving simple geometric shapes for others.

This interplay of the lattice and the associated dilation matrix, added to the fact that multidimensional filters are hard to design because of the absence of factorization theorems, makes the construction of multidimensional nonseparable regular wavelets much more difficult than in one 


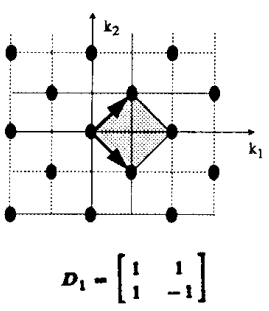

(a)
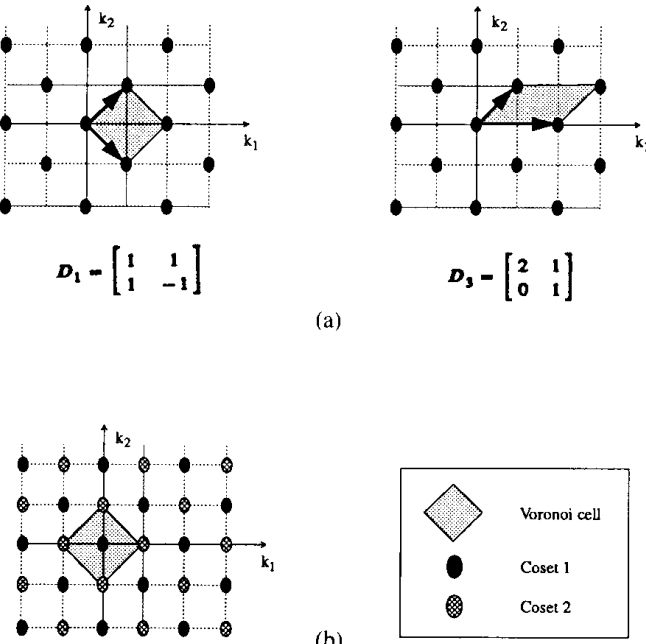

(b)

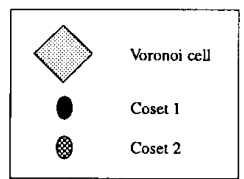

Fig. 2. (a) Quincunx lattice generated using two different matrices. Shaded regions represent fundamental parallelepipeds for each case. (b) Quincunx lattice with its Voronoi cell and cosets.

dimension. For example, a particular filter could be regular with respect to $D_{1}$, but not with respect to $D_{2}$ (see [7] for examples).

Thus, we see that while discrete signal processing is only concerned with the lattice, wavelet construction is concerned with the particular dilation matrix used to represent the lattice.

The present paper is concerned both with perfect reconstruction filter banks in multiple dimensions and with wavelets that can be obtained when iterating such filter banks. Therefore, we will have to develop the background for both subjects. While this leads to a few parts in the paper which are of a review or tutorial nature, it adds the benefit of a self-contained presentation. We will use both $z$-transform and Fourier domain notation, depending on which one is more appropriate. For example, synthesis of filter banks uses $z$-transforms, while discussion on wavelets is done in Fourier domain. While it is easy to go from one to the other, we sometimes indicate both next to each other.

Let us indicate below the principal results of the paper, along with an outline. Section II presents sampling on lattices, as well as sampling rate changes (the fundamental operations in multirate filter banks). Section III describes multidimensional multirate filter banks. There, we prove conditions for shift-invariance (so-called aliasing cancellation) and perfect reconstruction, in particular for the FIR case. We consider also orthogonal and linear phase (symmetric/antisymmetric filters) filter banks and their associated properties. Finally, separability of filters and of their polyphase components (the impulse responses on cosets of a lattice) is considered, showing possible alternatives. Section IV presents an extensive treatment of the two-channel case in multiple dimensions. This thorough treatment is done because it is the most natural generalization of the onedimensional case. The form of a general unitary polyphase matrix is given, as well as an analysis of possible linear phase solutions. We show that, as in one dimension, orthogonality and linear phase (symmetry) are exclusive for the real compact support (FIR) case. Section V addresses the synthesis of filter banks and proposes attractive cascade structures for the two-channel multidimensional case satisfying particular design constraints (such as orthogonality or linear phase). A general construction for small size linear phase filters is given for an arbitrary number of dimensions. For the fourchannel two-dimensional case we show how to obtain cascades leading to filter banks that are orthogonal and have linear phase [22], a solution which is impossible using separable filters. The transformation of one-dimensional filter banks into multidimensional ones is also described. In particular, the McClellan transformation, which can be used for biorthogonal filter banks, is discussed. Section VI develops the connection to nonseparable wavelet bases. First, the necessity of zeros at aliasing (or repeat) frequencies in the filters that are iterated is shown. Then, assuming that the iterated low-pass filter converges to a limit function in $L^{2}$, it is shown that all properties of one-dimensional wavelet bases generalize to higher dimensions, such as two-scale equations, orthogonality, and the fact that the wavelets form a basis for $L^{2}\left(\mathscr{R}^{n}\right)$ functions. Section VII shows some designs of orthogonal and biorthogonal wavelet bases, with numerical experiments indicating the conjectured regularity of the wavelet. Note that we do not address the regularity issue in any depth, and refer to the recent manuscript by Cohen and Daubechies [7] for a thorough treatment of the subject. For the sake of conciseness of the main text, most of the proofs are left to the Appendices, together with the definitions and notations.

Throughout the paper we will be dealing with a general multidimensional case. But at the end of each section the quincunx case will be used as an example to summarize all the important results of that section. Thus, the reader might go through this case study (Sections II-A, III-E, IV-C, V-C, VI-E, as well as Section VII on design) and get an overall idea of the basic concepts and results.

\section{Sampling in Multiple Dimensions}

In this section, some concepts and notions from the theory of lattices [5], [13] that will be used in the remainder of the paper are reviewed. Consider an analysis/synthesis filter bank as shown in Fig. 1. As pointed out in the Introduction, the two basic operations performed are filtering and sampling. The sampling process in $n$ dimensions can be represented by a lattice defined as the set of all linear combinations of $n$ basis vectors $a_{1}, a_{2}, \cdots, a_{n}$ with integer coefficients [5], [13], i.e., a lattice is the set of all vectors generated by $D k, k \in \mathscr{Z}^{n}$, where $D$ is the matrix characterizing the sampling process (its columns are the basis vectors $a_{1}, a_{2}, \cdots, a_{n}$ ). Since the elements of $D$ belong to $\mathscr{Z}$ which is a principal ideal ring, then unimodular matrices would be all those with determinant equal to \pm 1 [27]. Note that $D$ is not unique for a given sampling pattern and that two matrices representing the same sampling process are related by a linear transformation represented by a unimodular matrix [5]. A separable lattice is a lattice that can be represented by a 
diagonal matrix; it will appear when one-dimensional systems are used in a separable fashion along each dimension. The number of input lattice samples contained in the unit cell (the set of points such that disjoint union of its copies shifted to all of the lattice points yields the input lattice) represents the reciprocal of the sampling density and is given by $N=\operatorname{det} D$. An important unit cell is the fundamental parallelepiped $\mathscr{U}_{c}$ (the parallelepiped formed by $n$ basis vectors). In what follows, $\mathscr{U}_{c}^{t}$ will denote the fundamental parallelepiped of the transposed lattice. Shifting the origin of the output lattice to any of the points of the input lattice yields a so-called coset. Clearly, there are exactly $N$ distinct cosets obtained by shifting the origin of the output lattice to all of the points of the fundamental parallelepiped. The union of all cosets for a given lattice yields the input lattice.

Another important notion is that of the reciprocal lattice [5], [13]. This lattice is actually the Fourier transform of the original lattice and its points represent the points of replicated spectra in the frequency domain. If the matrix corresponding to the reciprocal lattice is denoted by $D_{r}$, then $D_{r}^{t} \cdot D=A$. $I$ and det $D \cdot \operatorname{det} D_{r}=A^{n}$. Now observe that the determinant of the matrix $D$ represents the hypervolume of any unit cell of the corresponding lattice, as well as the reciprocal of the sampling density. One of the possible unit cells is the Voronoi cell which is actually the set of points closer to the origin than to any other lattice point. The meaning of the unit cell in the frequency domain is extremely important since if the signal to be sampled is bandlimited to that cell, no overlapping of spectra will occur and the signal can be reconstructed from its samples.

To conclude the discussion on multidimensional sampling let us examine some operations involving sampling that are going to be used later. First, downsampling will mean that the points on the sampling lattice are kept while all the others are discarded. The time, Fourier, and $z$-domain expressions for the output of a downsampler are given by [13], [46]

$$
\begin{aligned}
y(n) & =x(D n), \\
\hat{Y}(\omega) & =\frac{1}{N} \sum_{k \in \mathscr{H}_{c}^{t}} \hat{X}\left(\left(D^{t}\right)^{-1} \cdot(\omega-2 \pi k)\right), \\
Y(z) & =\frac{1}{N} \sum_{k \in \mathscr{H}_{c}^{t}} X\left(W_{D^{-1}}(2 \pi k) \circ z^{D^{-1}}\right)
\end{aligned}
$$

where $N=\operatorname{det} D, \omega$ is an $n$-dimensional real vector, $z$ is an $n$-dimensional complex vector, and $n, \boldsymbol{k}$ are $n$-dimensional integer vectors (the details of the notation are defined in Appendix A). Next, consider upsampling, i.e., the process that maps a signal on the input lattice to another one that is nonzero only at the points of the sampling lattice

$$
\begin{aligned}
y(n) & =\left\{\begin{array}{cl}
x\left(D^{-1} n\right) & \text { if } \boldsymbol{n}=\boldsymbol{D} \boldsymbol{k} \\
0 & \text { otherwise, }
\end{array}\right. \\
\hat{Y}(\boldsymbol{\omega}) & =\hat{X}\left(\boldsymbol{D}^{t} \boldsymbol{\omega}\right), \\
Y(z) & =X\left(z^{D}\right) .
\end{aligned}
$$

Finally, combining (3) and (4), one obtains the expression for the output of a downsampler followed by an upsampler (that is, replacing by zeros all the samples that are not on the sublattice)

$$
\begin{aligned}
y(n) & =\left\{\begin{array}{cc}
x(n) & \text { if } n=D k \\
0 & \text { otherwise, }
\end{array}\right. \\
\hat{Y}(\omega) & =\frac{1}{N} \sum_{\boldsymbol{k} \in \mathbb{Z}_{c}^{t}} \hat{X}\left(\omega-2 \pi\left(D^{t}\right)^{-1} \boldsymbol{k}\right), \\
Y(z) & =\frac{1}{N} \sum_{\boldsymbol{k} \in \mathbb{Z}_{c}^{t}} X\left(W_{D^{-1}}(2 \pi k) \circ z\right) .
\end{aligned}
$$

\section{A. Quincunx Case}

The reason the quincunx case is examined in detail is because it uses the simplest multidimensional sampling structure that is nonseparable. This is obvious from Fig. 2(a) where the same lattice is generated using two sets of basis vectors (corresponding to matrices $D_{1}$ and $D_{2}$ given in (2)). Since the determinant of either one of them equals 2 , the corresponding critically sampled filter bank will have two channels. The same figure shows the fundamental parallelepipeds for both cases as well as the Voronoi cell. Since the reciprocal lattice for this case is again quincunx, its Voronoi cell will have the same diamond shape. This fact has been used in some image and video coding schemes [2], [43] since if restricted to this region; i) the spectra of the signal and its repeated occurrences that appear due to sampling will not overlap; and ii) due to the fact that the human eye is less sensitive to resolution along diagonals it is more appropriate for the low-pass filter to have diagonal cutoff. Note that the two vectors belonging to the unit cell are in both cases

$$
n_{0}=\left(\begin{array}{l}
0 \\
0
\end{array}\right), \quad n_{1}=\left(\begin{array}{l}
1 \\
0
\end{array}\right)
$$

and are the same for the unit cell of the transposed lattice, a fact that is going to be used throughout the paper. Shifting the origin of the quincunx lattice to points determined by the unit cell vectors yields the two cosets for this lattice (see Fig. 2(b)). Obviously, their union gives back the original lattice.

Finally, let us state here some facts that are going to be used later. First, following the notation in Appendix A, one can express $z^{D_{1}}$ as

$$
z^{D_{1}}=z^{\left(\begin{array}{rr}
1 & 1 \\
1 & -1
\end{array}\right)}=\left(z^{\left(\begin{array}{l}
1 \\
1
\end{array}\right)}, z^{\left(\begin{array}{r}
1 \\
-1
\end{array}\right)}\right)=\left(z_{1} z_{2}, z_{1} z_{2}^{-1}\right) .
$$

Then, using (4) one can write the expressions for the output of an upsampler in Fourier and $z$-domains

$$
\begin{aligned}
& \hat{Y}\left(\omega_{1}, \omega_{2}\right)=\hat{X}\left(\omega_{1}+\omega_{2}, \omega_{1}-\omega_{2}\right), \\
& Y\left(z_{1}, z_{2}\right)=X\left(z_{1} z_{2}, z_{1} z_{2}^{-1}\right) .
\end{aligned}
$$

Similarly, using (5), the output of a downsampler followed by an upsampler can be expressed as

$$
\begin{aligned}
& \hat{Y}\left(\omega_{1}, \omega_{2}\right)=\frac{1}{2}\left(\hat{X}\left(\omega_{1}, \omega_{2}\right)+\hat{X}\left(\omega_{1}+\pi, \omega_{2}+\pi\right)\right), \\
& Y\left(z_{1}, z_{2}\right)=\frac{1}{2}\left(X\left(z_{1}, z_{2}\right)+X\left(-z_{1},-z_{2}\right)\right) .
\end{aligned}
$$




\section{Multidimensional Perfect Reconstruction FILTER BANKS}

\section{A. Polyphase and Modulation Domain Analysis}

Consider a simple system consisting of a filter with the impulse response $h(0)=1, h(1)=2, h(2)=1,0$ otherwise, and of a downsampler by 2 (see Fig. 3). Then the impulse at time $0(x(n)=\delta(n))$ will produce the output $y(0)=1, y(1)=1,0$ otherwise, while an impulse at time 1 $(x(n)=\delta(n-1))$ will produce the output $y(1)=2,0$ otherwise. It is then obvious that the system, due to downsampling, is periodically shift-variant and for it to be completely specified one needs two impulse responses. It is this shiftvariance that leads to aliased versions (or overlapping repeated spectra) of the input signal in the output. A convenient way to take care of the shift-variance of such a multidimensional multirate system is to decompose both signals and filters into so-called polyphase components, each one corresponding to one of the cosets of the output lattice.

Then define the polyphase decomposition of the input signal as

$$
\begin{aligned}
X(z) & =\sum_{k \in \mathbb{Z}_{c}^{\prime}} z^{k} X_{k}\left(z^{D}\right)=p_{i}^{t}(z) \cdot x_{p}\left(z^{D}\right), \\
X_{k}(z) & =\sum_{n \in Z^{n}} x(D n-k) \cdot z^{-n}
\end{aligned}
$$

where $p_{i}(z)=\left\{z^{k}\right\}_{k \in \mathscr{H}_{c}^{t}}$ is a so-called vector of the inverse polyphase transform and $\boldsymbol{x}_{p}(z)$ is the vector containing the polyphase components of the input signal $x_{p}(z)=$ $\left\{X_{k}(z)\right\}_{k \in \ddot{H}_{c}^{t}}$. Note that the vector $p_{i}(z)$ is noncausal and its causal version will be denoted by $p_{i c}(z)$. Similarly, define the polyphase components of the filter $H(z)$ as

$$
\begin{aligned}
H(z) & =\sum_{k \in \|_{c}^{!}} z^{-k} H_{k}\left(z^{D}\right)=p_{f}^{t}(z) \cdot h_{p}\left(z^{D}\right), \\
H_{k}(z) & =\sum_{n \in Z^{n}} h(D n+k) \cdot z^{-n}
\end{aligned}
$$

where $p_{f}(z)=\left\{z^{-k}\right\}_{k \in \not y_{c}^{\prime}}$ is the vector of the forward polyphase transform and $h_{p}(z)$ is the vector containing the polyphase components of the filter, $h_{p}(z)=$ $\left\{H_{k}(z)\right\}_{k \in y_{c}^{\prime}}$. Note that the polyphase components of signals and filters are defined in a reverse fashion so as to account for the action of convolution. Therefore, a single-input linear periodically shift-variant system can be expressed as a multiinput linear shift-invariant system (for an example, see Fig. 3). To summarize, signals at the output of the analysis bank can be represented in terms of the input signal, forward polyphase transform $p_{f}(z)$, and the analysis polyphase matrix $H_{p}(z)$ (that is, the matrix containing the polyphase components of the analysis filters), while the output signal can be represented in terms of the input channel signals, the synthesis polyphase matrix $G_{p}(z)$ (that is, the matrix containing the polyphase components of the synthesis filters, defined in a reverse order from the analysis polyphase matrix) and the inverse polyphase transform $p_{i}(z)$ (see Fig. 4). Then, the output of the synthesis bank is
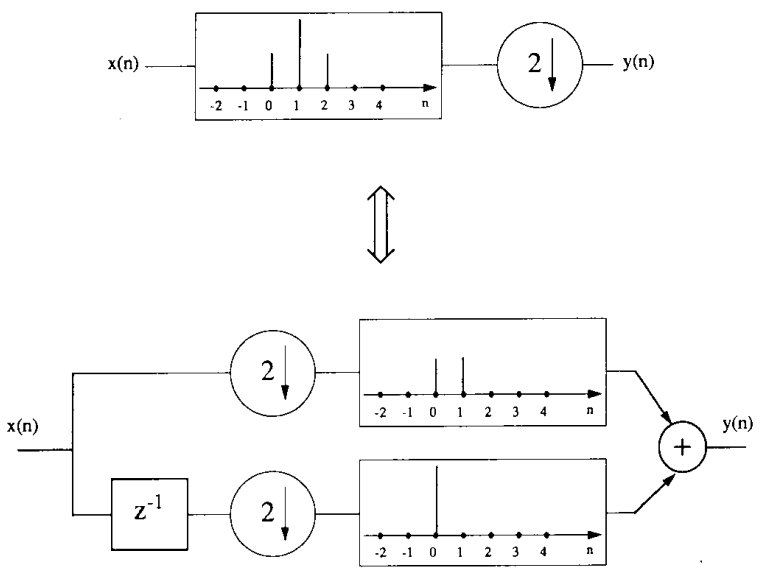

Fig. 3. Equivalent representations of a filter.

$$
\begin{aligned}
Y(z) & =p_{i c}^{t}(z) \cdot G_{p}\left(z^{D}\right) \cdot H_{p}\left(z^{D}\right) \cdot x_{p}\left(z^{D}\right), \\
& =p_{i c}^{t}(z) \cdot T_{p}\left(z^{D}\right) \cdot x_{p}\left(z^{D}\right)
\end{aligned}
$$

where $\boldsymbol{T}_{p}(\boldsymbol{z})=\boldsymbol{G}_{p}(\boldsymbol{z}) \boldsymbol{H}_{p}(\boldsymbol{z})$ is a so-called transfer polyphase matrix. The last equation yields easily results on alias cancellation and perfect reconstruction.

Lemma 3.1: 1) Aliasing is cancelled if and only if the inverse polyphase transform vector $\boldsymbol{p}_{i}$ is the left eigenvector of the transfer polyphase matrix in the upsampled domain $T_{p}\left(z^{D}\right)$, i.e., if and only if $p_{i}^{t} \cdot T_{p}\left(z^{D}\right)=T(z) \cdot p_{i}^{t}$, where $T(z)$ is the corresponding eigenvalue.

2) Perfect reconstruction is achieved if and only if the eigenvalue $T(z)$ associated with the eigenvector $\boldsymbol{p}_{i}^{t}$ in 1) is a monomial, i.e., if and only if $T(z)=c \cdot z^{-k}$.

3) Perfect reconstruction with FIR filters is achieved if and only if the determinant of the analysis polyphase matrix is a monomial, i.e., if and only if $\operatorname{det} \boldsymbol{H}_{p}(\boldsymbol{z})=\boldsymbol{z}^{-\boldsymbol{k}}$.

Proof: See Appendix B. Similar results have already appeared in [21], [45], [46].

The approach taken until now was to decompose signals and filters in such a way so that one can analyze the system as if it were shift-invariant. Here we proceed in a different manner, namely modulated versions of signals and filters are going to be used. Which approach is going to be applied to which problem depends on the nature of the problem itself. In the following sections both polyphase and modulation analysis will be employed intermittently.

The input signal and its modulated versions are given by

$$
\begin{aligned}
& \hat{\boldsymbol{x}}_{m}(\boldsymbol{\omega})=\left\{\hat{X}\left(\boldsymbol{\omega}-2 \pi\left(\boldsymbol{D}^{\prime}\right)^{-1} \boldsymbol{k}\right)\right\}_{\boldsymbol{k} \in \mathscr{H}_{c}^{\prime}}, \\
& \boldsymbol{x}_{m}(\boldsymbol{z})=\left\{X\left(W_{\boldsymbol{D}^{-1}}(2 \pi \boldsymbol{k}) \circ z\right)\right\}_{k \in \mathscr{W}_{c}^{t}}
\end{aligned}
$$

with notation as defined in Appendix A. Thus, the output of the system after upsampling and filtering in the synthesis 


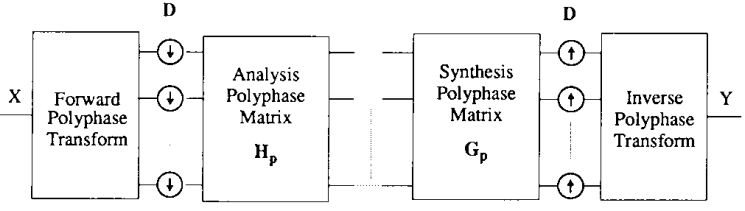

Fig. 4. Analysis/synthesis filter bank in the polyphase domain.

bank can be written as

$$
\begin{aligned}
\hat{Y}(\omega) & =\frac{1}{N}\left(\hat{G}_{0}(\omega) \cdots \hat{G}_{N-1}(\omega)\right) \cdot \hat{\boldsymbol{H}}_{m}(\omega) \cdot \hat{\boldsymbol{x}}_{m}(\omega), \\
Y(z) & =\frac{1}{N}\left(G_{0}(z) \cdots G_{N-1}(z)\right) \cdot \boldsymbol{H}_{m}(z) \cdot \boldsymbol{x}_{m}(z)
\end{aligned}
$$

where $\hat{H}_{m}(\omega), \boldsymbol{H}_{m}(\boldsymbol{z})$ contain the modulated versions of all the filters, that is

$$
\begin{aligned}
\hat{H}_{m}(\omega)= & \left\{\hat{H}_{i}\left(\omega-2 \pi\left(D^{t}\right)^{-1} k\right)\right\}, \\
H_{m}(z)= & \left\{H_{i}\left(W_{D^{-1}}(2 \pi k) \circ z\right)\right\}, \\
& k \in \mathscr{U}_{c}^{t}, \quad i \in\{0, \cdots, N-1\} .
\end{aligned}
$$

Let us note at this point that the relationship between polyphase and modulation domain analysis is in a way like "time-Fourier" domain relation; hence the two representations are related by Fourier transforms. For more details, see [39].

After having achieved the goal of obtaining perfect reconstruction one might impose some other requirements on the filter bank, some of the important ones being that the bank be orthogonal and/or linear phase. Note that when compared to the one-dimensional case the linear phase requirement is less constrained since linear phase in multiple dimensions means just centro-symmetry of the filter's impulse response [14].

\section{B. Orthogonal Case}

A filter with a rational transfer function is called allpass if it satisfies

$$
\tilde{H}(z) \cdot H(z)=1
$$

while a square matrix with rational entries is called paraunitary if it satisfies

$$
\tilde{H}(z) \cdot \boldsymbol{H}(z)=\boldsymbol{H}(z) \cdot \tilde{H}(z)=c \cdot \boldsymbol{I} .
$$

On the unit hypercircles $\left(z_{i}=e^{j \omega_{i}}, i=1, \cdots, n\right)$ this matrix becomes orthogonal, and for the sake of simplicity it will be referred to as such throughout the paper. Now suppose that the analysis polyphase matrix is orthogonal. Then by choosing $\boldsymbol{G}_{p}(z)=z^{-\boldsymbol{k}} \tilde{\boldsymbol{H}}_{p}(z)$, a perfect reconstruction system is obtained as can be seen from (12). This solution has important advantages both from the theoretical point of view (the filter bank calculates projections on orthogonal subspaces) and from the point of view of implementation (the synthesis filters are within shift-reversal the same as the analysis ones).
Suppose now that the modulation matrix defined in (15) is orthogonal. Then using (17) (with $c=N$ )

$$
\sum_{\boldsymbol{k} \in \mathbb{W}_{c}^{!}} H_{i}\left(W_{D^{-1}}(2 \pi k) \circ z\right) \tilde{H}_{j}\left(W_{D^{-1}}(2 \pi k) \circ z\right)=N \cdot \delta_{i j} .
$$

It is obvious that by choosing $\left(G_{0}(z) \cdots G_{N-1}(z)\right)$ as the first row of $\tilde{\boldsymbol{H}}_{m}(z)$, perfect reconstruction is achieved as can be seen from (14). Now observe that assuming real coefficients, $H_{i}(z) \tilde{H}_{j}(z)$ is the $z$-transform of the crosscorrelation sequence $r_{i j}(\boldsymbol{n})=\left\langle h_{i}(\boldsymbol{k}), h_{j}(\boldsymbol{k}+\boldsymbol{n})\right\rangle$. Also note that $H_{i}\left(a_{1} z_{1}, \cdots, a_{n} z_{n}\right) \tilde{H}_{j}\left(a_{1} z_{1}, \cdots, a_{n} z_{n}\right)$ is the $z$ transform of $a_{1}^{-n_{1}} \cdots \cdots a_{n}^{-n_{n}} \cdot r_{i j}\left(n_{1}, \cdots, n_{n}\right)$. Using these facts one can see that (18) is the $z$-transform of

$$
r_{i j}(n) \cdot \sum_{k \in \|_{c}^{l}} e^{j 2 \pi k^{\prime} D^{-1} n}=N \cdot \delta_{i j} \delta_{n} .
$$

Analogously to the one-dimensional case, it can be shown that the sum in the previous equation is nonzero only at lattice points [34]

$$
\sum_{k \in \|_{c}^{t}} e^{j 2 \pi k^{\prime} D^{-1} n}=\left\{\begin{array}{lc}
N & \text { if } n=D n_{0} \\
0 & \text { otherwise. }
\end{array}\right.
$$

Using this fact, one can finally write (19) as

$$
r_{i j}(D n)=\left\langle h_{i}(\boldsymbol{k}), h_{j}(\boldsymbol{k}+\boldsymbol{D n})\right\rangle=\delta_{i j} \delta_{n}
$$

showing that each filter is orthogonal to its translates with respect to the lattice in question, and pairs of filters are orthogonal to each other and their shifts with respect to the lattice, that is, the set $S=\left\{h_{i}(k+D n) \mid i=0, \cdots\right.$, $\left.N-1, k, n \in \mathscr{Z}^{n}\right\}$ is an orthonormal set. This is the lattice extension of the well-known orthogonality relations with respect to shifts in the one-dimensional case [9], [41].

\section{Linear Phase Case}

Let us begin this section by introducing some notation. Suppose we circumscribe a parallelepiped around a polynomial represented in the space of its exponents. To facilitate the discussion (since we are interested in $z$-transform), a point $(m, n)$ in this space will denote the polynomial term $z_{1}^{-m} z_{2}^{-n}$, i.e., the exponents will be taken with a minus sign. Then define $\boldsymbol{P}=\left(p_{1}, p_{2}, \cdots, p_{n}\right)$ and $\boldsymbol{Q}=\left(q_{1}\right.$, $q_{2}, \cdots, q_{n}$ ) as the corners on the main hyperdiagonal of the parallelepiped. The size of the polynomial in the $i$ th direction is $l_{i}=q_{i}-p_{i}+1$. When dealing with more than one polynomial at a time, their $P$ 's and $Q$ 's will be distinguished by a superscript. For example, $\boldsymbol{P}^{(1)}$ is $\boldsymbol{P}$ of the first polynomial, while $p_{2}^{(1)}$ is the second coordinate of $\boldsymbol{P}$ of the first polynomial. Fig. 5 shows the above notation on an example in two dimensions.

The aim here is to derive a condition in terms of the analysis polyphase matrix that can be used to test linear phase of all the filters in the bank simultaneously. If a real filter is linear phase then it can be written as

$$
H(z)=a \cdot D(z) \cdot \tilde{H}(z)
$$




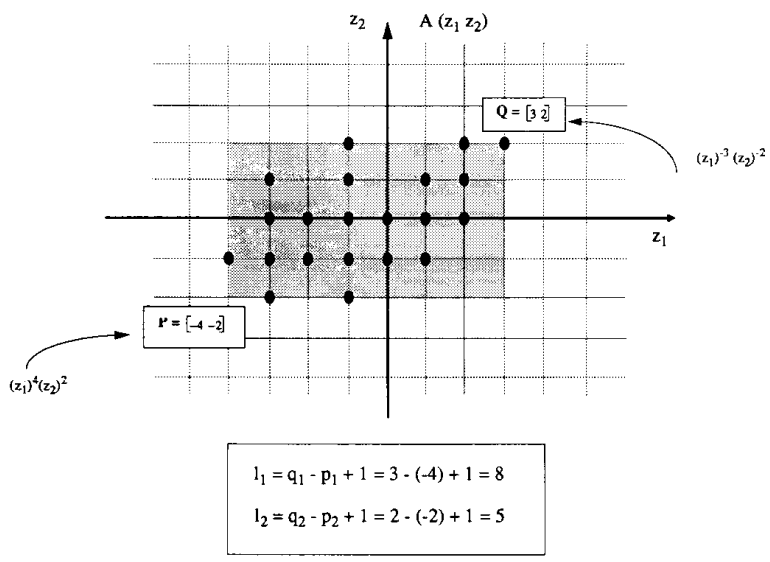

Fig. 5. Notation used for polynomials. $P$ and $Q$ denote the corners of the parallelogram circumscribed around the polynomial. It is represented in the space of its exponents. $l_{i}$ denotes the length of the polynomial in the $i$ th direction.

where $a$ denotes the symmetry of the filter ( 1 for symmetric, -1 for antisymmetric) and

$$
D(z)=z^{-(P+Q)}=\prod_{i=1}^{n} z_{i}^{-\left(p_{i}+q_{i}\right)}
$$

with $P$ and $Q$ as defined above. A similar relation can be defined for the analysis polyphase matrix. Following the discussion in Section III-A one has that the vector containing the analysis filters can be written as

$$
h(z)=\left(H_{0}(z) \cdots H_{N-1}(z)\right)^{t}=H_{p}\left(z^{D}\right) \cdot p_{f}(z)
$$

Suppose now that all of the filters are linear phase. Then each one can be expressed as in (22), and thus,

$$
\begin{aligned}
\boldsymbol{H}_{p}\left(z^{D}\right) \cdot p_{f}(z) & \left(\begin{array}{c}
H_{0}(z) \\
\vdots \\
H_{N-1}(z)
\end{array}\right)=\left(\begin{array}{c}
a_{0} \cdot D_{0}(z) \cdot \tilde{H}_{0}(z) \\
\vdots \\
a_{N-1} \cdot D_{N-1}(z) \cdot \tilde{H}_{N-1}(z)
\end{array}\right), \\
= & \left(\begin{array}{c}
a_{0} \cdot \\
\cdot a_{N-1}
\end{array}\right)\left(\begin{array}{c}
D_{0}(z) \\
\ddots \\
D_{N-1}(z)
\end{array}\right)\left(\begin{array}{c}
\tilde{H}_{0}(z) \\
\vdots \\
\tilde{H}_{N-1}(z)
\end{array}\right), \\
= & \left(\begin{array}{c}
a_{0} \cdot \\
\cdot a_{N-1}
\end{array}\right)\left(\begin{array}{c}
D_{0}(z) \\
D_{N-1}(z)
\end{array}\right) \\
& \cdot \tilde{H}_{p}^{t}\left(z^{D}\right) \cdot \tilde{p}_{f}^{t}(z),=a \cdot \Delta(z) \cdot \tilde{H}_{p}^{t}\left(z^{D}\right) \cdot \tilde{p}_{f}^{t}(z) .
\end{aligned}
$$

This can be used as a linear phase testing condition for the whole filter bank. Note that $\tilde{p}_{f}^{t}(z)=\boldsymbol{p}_{i}(\boldsymbol{z})$. Basically (23) allows 1) to check linear phase of an already designed filter bank; or 2) if one wishes to design a filter bank containing linear phase filters, one can impose constraints on the polyphase matrix in such a way that (23) is satisfied. Another linear phase testing condition appeared in [21].

\section{Separability Versus Nonseparability}

As already noted in Section II, sampling lattices that are used can be both separable and nonseparable ones. In both cases an interesting question is whether it is possible to have all combinations separable/nonseparable sampling/filters/ polyphase components.

To analyze separable sampling, let us first try to determine the number of free variables when the filters and polyphase components are separable/nonseparable. If a filter is separable it has $F V_{1}=\sum_{i=1}^{n}\left(l_{i}-1\right)$ free variables where $l_{i}$, $i=1, \cdots, n$ is the size of the filter in the $i$ th dimension. The reason that 1 is subtracted in each term is because of scaling. If, on the other hand, the filter is nonseparable, the number of free variables it possesses is $F V_{2}=\Pi_{i=1}^{n} l_{i}-1$. Now, what happens if the polyphase components are separable? First note that if the sampling factor in the $i$ th dimension is $N_{i}$, then the size of the polyphase component $H_{i_{1} i_{2} \cdots i_{n}}$, $i_{j}=1, \cdots, N_{j}$ in the $i$ th dimension can be expressed as $l_{i_{1}, \cdots, i_{n} j}=\left(l_{j}+N_{j}-i_{j}\right)$ mod $N_{j}$. Thus, the number of free variables if the polyphase components are separable is $F V_{3}=\sum_{i_{1}, \cdots, i_{n}}\left(\sum_{j} l_{i_{1} \cdots i_{n} j}-n+1\right)-1$ which after some manipulations can be written as $F V_{3}=\Pi_{j=1}^{n}$ $N_{j}\left(\sum_{i=1}^{n}\left(l_{i} / N_{i}\right)-(n-1)\right)-1$. This number is in general different from $F V_{1}$ and $F V_{2}$ and satisfies the following:

$$
F V_{1}<F V_{3} \leq F V_{2}
$$

In the above, equality holds just for very small filter sizes (basically when all polyphase components are either constants or one-dimensional polynomials since in that case there is no distinction between separable and nonseparable polyphase components). Thus, one may conclude that in general separable polyphase components would yield a nonseparable filter (in other words to get a separable filter assuming separable polyphase components one would have to reduce the number of free variables). Note that the assumed size of the filter in each dimension is at least two. Finally, if polyphase components are nonseparable the number of free variables is $F V_{4}$ $=\sum_{i_{1}, \cdots, i_{n}} \Pi_{j=1}^{n} l_{i_{1} \cdots i_{n} j}-1=\Pi_{j=1}^{n} \sum_{i_{j}}\left(\left(l_{j}+N_{j}-i_{j}\right) \bmod \right.$ $\left.N_{j}\right)-1 \stackrel{1}{=} \Pi_{i=1}^{n} l_{i}-1=F V_{2}$, that is, if the polyphase components are nonseparable then the total number of free variables is the same as when the filter itself is nonseparable

$$
F V_{4}=F V_{2}
$$

Fact 3.1: For separable sampling, the following holds:

1) separable filter implies separable polyphase components;

2) nonseparable polyphase components will always yield a nonseparable filter. 
Proof:

1) A separable filter can be expressed as follows:

$$
\begin{aligned}
& H\left(z_{1}, \cdots, z_{n}\right) \\
& =H^{(1)}\left(z_{1}\right) \cdots H^{(n)}\left(z_{n}\right), \\
& =\sum_{i_{1}=0}^{N_{1}-1} z_{1}^{-i_{1}} H_{i_{1}}^{(1)}\left(z_{1}^{N_{1}}\right) \cdots \sum_{i_{n}=0}^{N_{n}-1} z_{n}^{-i_{n}} H_{i_{n}}^{(n)}\left(z_{n}^{N_{n}}\right), \\
& =\sum_{i_{1}=0}^{N_{1}-1} \cdots \sum_{i_{n}=0}^{N_{n}-1} z_{1}^{-i_{1}} \cdots z_{n}^{-i_{n}} \cdot H_{i_{1}}^{(1)}\left(z_{1}^{N_{1}}\right) \cdots H_{i_{n}}^{(n)}\left(z_{n}^{N_{n}}\right)
\end{aligned}
$$

where $H_{i_{1}}^{(1)}\left(z_{1}\right) \cdots \cdots H_{i_{n}}^{(n)}\left(z_{n}\right)$, corresponding to the polyphase components of the filter, are clearly separable.

2) Due to (25).

For nonseparable sampling, things become more complicated. Consider, for example, what happens if the filter is separable. Writing it in terms of its polyphase components it is obvious that to get separable polyphase components one would have to reduce the number of degrees of freedom. It follows similarly for the nonseparable filter. Thus, in general both separable and nonseparable filters would have nonseparable polyphase components. On the other hand, if the polyphase components are separable, after upsampling they would in general become nonseparable, yielding in turn a nonseparable filter. For nonseparable polyphase components this is even more obvious. Therefore, starting from either separable or nonseparable polyphase components one would obtain a nonseparable filter. Since in this case, there are no implications as for separable sampling one can find examples for any combination separable/nonseparable filter/polyphase components. However, in general (that is, except for particular values of coefficients) one has that separable polyphase components do not yield separable filters and separable filters do not yield separable polyphase components.

\section{E. Quincunx Case Revisited}

Let us continue our analysis of the quincunx case. As we said in Section III-A, polyphase domain analysis is used to help overcome problems that arise when dealing with shiftvariant systems. Thus, in this case polyphase decomposition would correspond to considering the system "living" on the two cosets of the quincunx lattice (see Fig. 2(b)). For example, if a filter's impulse response is denoted by $h(n)=$ $h\left(n_{1}, n_{2}\right)$ its two polyphase components can be written as (matrix $D_{1}$ from (2) is used)

$$
\begin{aligned}
& h_{0}\left(n_{1}, n_{2}\right)=h(\boldsymbol{D n})=h\left(n_{1}+n_{2}, n_{1}-n_{2}\right), \\
& h_{1}\left(n_{1}, n_{2}\right)=h\left(\boldsymbol{D n}+n_{1}\right)=h\left(n_{1}+n_{2}+1, n_{1}-n_{2}\right),
\end{aligned}
$$

where $n_{1}$ (see (6)) belongs to the unit cell (in this case to the unit cell of the transposed lattice as well) and is the representative vector of one of the cosets. From (8), (26), and (27) it is obvious that the $z$-transform expression of a filter is then

$$
\begin{aligned}
H\left(z_{1}, z_{2}\right) & =H_{0}\left(z^{D}\right)+z_{1}^{-1} H_{1}\left(z^{D}\right) \\
& =H_{0}\left(z_{1} z_{2}, z_{1} z_{2}^{-1}\right)+z_{1}^{-1} H_{1}\left(z_{1} z_{2}, z_{1} z_{2}^{-1}\right) .
\end{aligned}
$$

The vectors of forward and inverse polyphase transforms (causal versions) being $p_{f}\left(z_{1}, z_{2}\right)^{t}=\left(1 z_{1}^{-1}\right)$ and $p_{i c}\left(z_{1}, z_{2}\right)^{t}=\left(z_{1}^{-1} 1\right)$, respectively (see Section III-A), one gets (12) for the quincunx case

$$
\begin{aligned}
Y\left(z_{1}, z_{2}\right)=\left(\begin{array}{ll}
z_{1}^{-1} & 1
\end{array}\right) \boldsymbol{G}_{p}\left(z_{1} z_{2}, z_{1} z_{2}^{-1}\right) \\
\cdot \boldsymbol{H}_{p}\left(z_{1} z_{2}, z_{1} z_{2}^{-1}\right) \boldsymbol{x}_{p}\left(z_{1} z_{2}, z_{1} z_{2}^{-1}\right)
\end{aligned}
$$

where the analysis polyphase matrix can be written as $\left(H_{i j}\right.$ denotes the $j$ th polyphase component of the $i$ th filter)

$$
H_{p}\left(z_{1}, z_{2}\right)=\left(\begin{array}{ll}
H_{00}\left(z_{1}, z_{2}\right) & H_{01}\left(z_{1}, z_{2}\right) \\
H_{10}\left(z_{1}, z_{2}\right) & H_{11}\left(z_{1}, z_{2}\right)
\end{array}\right) .
$$

To give a gist of the modulation domain analysis let us first find the modulated versions of the input signal as given in (13)

$$
\begin{aligned}
\boldsymbol{x}_{m}\left(z_{1}, z_{2}\right) & =\left\{X\left(\left(\begin{array}{l}
e^{0} \\
e^{0}
\end{array}\right) \circ\left(\begin{array}{l}
z_{1} \\
z_{2}
\end{array}\right)\right), X\left(\left(\begin{array}{l}
z_{1} \\
z_{2}
\end{array}\right) \circ\left(\begin{array}{c}
e^{-j \pi} \\
e^{-j \pi}
\end{array}\right)\right)\right\} \\
& =\left\{X\left(z_{1}, z_{2}\right), X\left(-z_{1},-z_{2}\right)\right\} .
\end{aligned}
$$

Then (14) for the output of the system is

$$
\begin{aligned}
& \hat{Y}\left(\omega_{1}, \omega_{2}\right)=\frac{1}{2}\left(\hat{G}_{0}\left(\omega_{1}, \omega_{2}\right) \hat{G}\left(\omega_{1}, \omega_{2}\right)\right) \\
& \cdot\left(\begin{array}{ll}
\hat{H}_{0}\left(\omega_{1}, \omega_{2}\right) & \hat{H}_{0}\left(\omega_{1}+\pi, \omega_{2}+\pi\right) \\
\hat{H}_{1}\left(\omega_{1}, \omega_{2}\right) & \hat{H}_{1}\left(\omega_{1}+\pi, \omega_{2}+\pi\right)
\end{array}\right) \\
& \cdot\left(\begin{array}{c}
\hat{X}\left(\omega_{1}, \omega_{2}\right) \\
\hat{X}\left(\omega_{1}+\pi, \omega_{2}+\pi\right)
\end{array}\right), \\
& Y\left(z_{1}, z_{2}\right)=\frac{1}{2}\left(G_{0}\left(z_{1}, z_{2}\right) G_{1}\left(z_{1}, z_{2}\right)\right) \\
& \cdot\left(\begin{array}{ll}
H_{0}\left(z_{1}, z_{2}\right) & H_{0}\left(-z_{1},-z_{2}\right) \\
H_{1}\left(z_{1}, z_{2}\right) & H_{1}\left(-z_{1},-z_{2}\right)
\end{array}\right) \\
& \cdot\left(\begin{array}{c}
X\left(z_{1}, z_{2}\right) \\
X\left(-z_{1},-z_{2}\right)
\end{array}\right) \text {. }
\end{aligned}
$$

Note the resemblance between this expression and its one-dimensional two-channel counterpart (see, for example, [9], [40]). This striking similarity that appears when analyzing the quincunx case time and again is basically due to the fact that a number of results depend heavily on the overall sampling density (number of channels for the critically sampled filter bank). Thus, as will be seen in Section IV, some of the results obtained for the classical one-dimensional two- 
channel filter bank will extend easily to the $n$-dimensional two-channel case.

To illustrate the statement that the polyphase and modulation domain representations are related through Fourier transform, consider the following relation between polyphase and modulation domain matrices:

$$
\begin{aligned}
\hat{\boldsymbol{H}}_{p}\left(\omega_{1}+\omega_{2}, \omega_{1}-\omega_{2}\right)= & \frac{1}{2} \hat{\boldsymbol{H}}_{m}\left(\omega_{1}, \omega_{2}\right) \\
& \cdot\left(\begin{array}{cc}
1 & 1 \\
1 & -1
\end{array}\right)\left(\begin{array}{cc}
1 & 0 \\
0 & e^{j \omega_{1}}
\end{array}\right), \\
\boldsymbol{H}_{p}\left(z_{1} z_{2}, z_{1} z_{2}^{-1}\right)= & \frac{1}{2} \boldsymbol{H}_{m}\left(z_{1}, z_{2}\right) \\
& \cdot\left(\begin{array}{rr}
1 & 1 \\
1 & -1
\end{array}\right)\left(\begin{array}{cc}
1 & 0 \\
0 & z_{1}
\end{array}\right) .
\end{aligned}
$$

Next, one can examine the relation between the two filters in the analysis bank if the system is orthogonal. Without going through the whole analysis from Section III-B just write (21) for the quincunx case ( $D$ represents quincunx sampling)

$$
\begin{gathered}
\left\langle h_{0}(k), h_{0}(k+D n)\right\rangle=\delta_{n}, \\
\left\langle h_{1}(k), h_{1}(k+D n)\right\rangle=\delta_{n}, \\
\left\langle h_{0}(k), h_{1}(k+D n)\right\rangle=0 .
\end{gathered}
$$

Equations (34) and (35) show that each filter is orthogonal to its shifts on the quincunx lattice, while (36) states that filters $h_{0}$ and $h_{1}$ are orthogonal to each other as well as their shifts on the quincunx lattice. These facts are going to be used when constructing orthonormal bases of wavelets for the quincunx case in Section VI.

Finally, let us show the linear phase testing condition given by (23) for the quincunx case

$$
\begin{gathered}
\boldsymbol{H}_{p}\left(z_{1} z_{2}, z_{1} z_{2}^{-1}\right)\left(\begin{array}{c}
1 \\
z_{1}^{-1}
\end{array}\right) \\
=\left(\begin{array}{cc}
a_{0} & 0 \\
0 & a_{1}
\end{array}\right)\left(\begin{array}{cc}
D_{0}\left(z_{1}, z_{2}\right) & 0 \\
0 & D_{1}\left(z_{1}, z_{2}\right)
\end{array}\right) \\
\cdot \boldsymbol{H}_{p}\left(z_{1}^{-1} z_{2}^{-1}, z_{1}^{-1} z_{2}\right)\left(\begin{array}{c}
1 \\
z_{1}
\end{array}\right) .
\end{gathered}
$$

Here $a_{0}$ and $a_{1}$ demonstrate what kind of a symmetry filters $h_{0}$ and $h_{1}$ possess ( 1 for symmetric and -1 for antisymmetric) and $D_{0}, D_{1}$ are monomials related to the size of the filter defined in Section III-C.

\section{Two-Channel Case In Multiple Dimensions}

Let us first point out that the two-channel case we are going to deal with in this section is the only true nonseparable one in terms of the sampling used. Among others, it can be characterized by one of the following sampling matrices:

$$
\begin{aligned}
& \boldsymbol{D}_{1}=\left(\begin{array}{ccccc}
1 & 0 & \cdots & 0 & 1 \\
1 & 1 & \cdots & 0 & 0 \\
0 & 1 & \cdots & 0 & 0 \\
\vdots & \vdots & \ddots & \vdots & \vdots \\
0 & 0 & \cdots & 1 & 0 \\
0 & 0 & \cdots & 1 & (-1)^{n-1}
\end{array}\right), \\
& \boldsymbol{D}_{2}=\left(\begin{array}{ccccc}
2 & 1 & 0 & \cdots & 0 \\
0 & 1 & 1 & \cdots & 0 \\
0 & 0 & 1 & \cdots & 0 \\
\vdots & \vdots & \vdots & \ddots & \vdots \\
0 & 0 & 0 & \cdots & 1 \\
0 & 0 & 0 & \cdots & 1
\end{array}\right)
\end{aligned}
$$

Since the overall sampling density is 2 , the equivalent sampling factor per dimension would be $2^{1 / n}$. In two dimensions the corresponding lattice is the quincunx lattice and in three dimensions the FCO or face centered orthorhombic lattice. Since they are natural extensions of the one-dimensional two-channel case they already found application in image and video processing [13], [19], [43].

Refer to the discussion on the modulation domain analysis in Section III-A. For the two-channel case the output of the system given by (14) can be written as

$$
\begin{aligned}
& Y(z)=\frac{1}{2}\left(G_{0}(z) G_{1}(z)\right) \cdot\left(\begin{array}{cc}
H_{0}(z) & H_{0}(-z) \\
H_{1}(z) & H_{1}(-z)
\end{array}\right) \\
& \cdot\left(\begin{array}{c}
X(z) \\
X(-z)
\end{array}\right)
\end{aligned}
$$

which, as already pointed out for the quincunx case in Section III-E, (32), bears striking resemblance to the onedimensional case. Then it is easy to see that the result on alias cancellation (or no overlapping of spectra) obtained for the one-dimensional case [8] holds for an arbitrary number of dimensions:

Proposition 4.1: The classical QMF solution holds for the $n$-dimensional filter bank, i.e., the following choice of filters will yield alias cancellation.

$$
\begin{array}{lll}
H_{0}(z)=H(z) & \text { and } & H_{1}(z)=H(-z) \\
G_{0}(z)=H(z) & \text { and } & G_{1}(z)=-H(-z) .
\end{array}
$$

As already pointed out in Section III additional constraints can be imposed upon the filters in the bank beside requiring perfect reconstruction. In what follows two cases of interest are investigated, the first one yielding orthogonal filters and the second linear phase ones. Note that in the two-channel real FIR case these requirements are mutually exclusive as will be shown later.

\section{A. Orthogonal Case}

If the filter bank is orthogonal it can be shown that the filters involved have some important structural properties, 
namely that a single filter specifies completely the most general orthogonal system. Here we just state the theorem and its corollaries, for the proofs refer to Appendix C.

Theorem 4.1: The most general $2 \times 2$ real FIR orthogonal polyphase matrix $\boldsymbol{H}_{p}$ can be written in the following form:

$$
H_{p}(z)=\left(\begin{array}{cc}
1 & 0 \\
0 & z^{-k}
\end{array}\right) \cdot\left(\begin{array}{cc}
H_{00}(z) & H_{01}(z) \\
c \tilde{H}_{01}(z) & -c \tilde{H}_{00}(z)
\end{array}\right)
$$

where $c$ is $\pm 1, k$ is large enough so as to make the entries in the second row causal, and $H_{00}$ and $H_{01}$ satisfy the PC property.

Corollary 4.1: The polyphase components of each filter are of the same size.

Corollary 4.2: The second filter is completely specified by modulating and reversing the first one, i.e.,

$$
H_{1}(z)=-z^{-k^{\prime}} \cdot \tilde{H}_{0}(-z)
$$

where

$$
\begin{aligned}
z^{-k^{\prime}}=z_{1}^{-\left(k_{1}+k_{n}+1\right)} z_{2}^{-\left(k_{1}+k_{2}\right)} & \\
& \cdots z_{n-1}^{-\left(k_{n-2}+k_{n-1}\right)} z_{n}^{-\left(k_{n-1}+(-1)^{n-1} k_{n}\right)} .
\end{aligned}
$$

\section{B. Linear Phase Solutions}

Which solutions are possible if one wants a perfect reconstruction system where both filters are linear phase? The first polyphase component of each filter will be denoted by $H_{i 0}$ and the second one by $H_{i 1}, i=1,2$. The whole analysis will be performed in the upsampled domain and thus the filters can be expressed as (see Section III-A and (28))

$$
H_{i}(z)=H_{i 0}\left(z^{D}\right)+z_{1}^{-1} H_{i 1}\left(z^{D}\right)=a_{i} \cdot D_{i}(z) \cdot \tilde{H}_{i}(z)
$$

where $a_{i}$ and $D_{i}(z)$ characterize a linear phase filter as defined in (22). The determinant of the polyphase matrix in the upsampled domain is then

$$
\begin{aligned}
\operatorname{det} H_{p}\left(z^{D}\right) \\
\quad=T\left(z^{D}\right) \\
\quad=H_{00}\left(z^{D}\right) H_{11}\left(z^{D}\right)-H_{10}\left(z^{D}\right) H_{01}\left(z^{D}\right)
\end{aligned}
$$

and the aim here is to force it to be a monomial in order to achieve perfect reconstruction.

The sizes of the filter $H_{i}$ will be denoted by $P^{(i)}, Q^{(i)}$ (where $P$ and $Q$ are as introduced in Section III-C). Then for the filters' polyphase components in the upsampled domain the following holds: $H_{i 0}$ is of size $P^{(i)}, Q^{(i)}$ and $H_{i 1}$ is of size $\boldsymbol{P}^{(i)}-(1,0, \cdots, 0), Q^{(i)}-(1,0, \cdots, 0)$. The determinant $T\left(z^{D}\right)$ is of size $P^{(T)}$ and $Q^{(T)}$, where $P^{(T)}=$ $\boldsymbol{P}^{(1)}+\boldsymbol{P}^{(2)}-(1,0, \cdots, 0), \quad \boldsymbol{Q}^{(T)}=Q^{(1)}+Q^{(2)}-(1$, $0, \cdots, 0)$ and $l_{k}^{(T)}=l_{k}^{(1)}+l_{k}^{(2)}-1$.

Here, we will just state some facts on the structure of linear phase solutions. For explicit proofs, refer to [19]. Note that $i(n)$ is \pm 1 if $n$ is even/odd, respectively.
Proposition 4.2: For a symmetric/antisymmetric filter the following holds:

$$
\begin{aligned}
& \text { 1) } i\left(\sum_{k=1}^{n} l_{k}\right)=(-1)^{n} \Leftrightarrow H_{i 0}\left(z^{D}\right)=a_{i} D_{i}(z) \tilde{H}_{i 0}\left(z^{D}\right) \\
& \text { and } H_{i 1}\left(z^{D}\right)=a_{i} D_{i}(z) z_{1}^{2} \tilde{H}_{i 1}\left(z^{D}\right), \\
& \text { 2) } i\left(\sum_{k=1}^{n} l_{k}\right)=(-1)^{n-1} \Leftrightarrow H_{i 1}\left(z^{D}\right)= \\
& a_{i} D_{i}(z) z_{1} \tilde{H}_{i 0}\left(z^{D}\right)
\end{aligned}
$$

where $a_{i}$ and $D_{i}(z)$ are associated with the filter as defined in (22).

This proposition basically states how the polyphase components of a symmetric/antisymmetric filter are related depending upon the size of the filter. For example, in one dimension it is easy to see that an odd length filter (case 1)) will have each polyphase component as a symmetric/ antisymmetric filter, while if the length is even (case 2)) one can get the second polyphase component by shift-reversing the first one. To obtain perfect reconstruction with FIR filters the determinant $T$ has to be a monomial (following Lemma 3.1). Now observe that taking all possible combinations of the two filters one can obtain $T$ being symmetric/ antisymmetric or $T$ that does not have any specific symmetry. In what follows just the former case will be considered since all useful solutions found until now belong to that category.

Proposition 4.3: For perfect reconstruction with a symmetric/antisymmetric determinant $T, T$ has to satisfy the following:

1) $i\left(l_{k}^{(r)}\right)=-1, \forall k$, i.e., $T$ is of odd size in all directions;

2) $a_{T}=1$, i.e., $T$ has to be symmetric;

3) $i\left(\sum_{k=1}^{n}\left(p_{k}^{(T)}+q_{k}^{(T)}\right) / 2\right)=1$, i.e., the degree of the center coefficient is even.

Proposition 4.4: For perfect reconstruction with a symmetric/antisymmetric $T$ and linear phase filters, there are two possible cases:

1) both filters are as in Proposition 4.2, case 1) and they have the same symmetry, i.e., $a_{1} a_{2}=1$; or

2) both filters are as in Proposition 4.2, case 2) and they have different symmetry, i.e., $a_{1} a_{2}=-1$.

Proposition 4.5: For a perfect reconstruction linear phase filter pair the following holds:

1) it is not possible to have the filters of the same symmetry and the same size;

2) It is possible to have the filters of opposite symmetry and the same size.

As an example consider the one-dimensional case. There, it has been shown that assuming a symmetric/antisymmetric $T$ either both filters are of odd length and the same symmetry or they are of even length and opposite symmetry (the uninteresting case has not been considered) [42].

Proposition 4.6: In the two-channel real FIR case linear phase and orthogonality requirements are mutually exclusive (except for the trivial two-tap filters). 
Proof: To prove this, it will be shown that it is not possible to have a linear phase filter whose polyphase components satisfy the PC property as required by Theorem 4.1 . Since by Corollary 4.1 the polyphase components of each filter are of the same size, i.e., the filters are of the same size, then they have to have opposite symmetry and they belong to case 2) of Proposition 4.2 (by the previous discussion). Thus, substituting polyphase components into (90) one obtains

$$
\begin{aligned}
H_{i 0}\left(z^{D}\right) \tilde{H}_{i 0}\left(z^{D}\right)+H_{i 1}\left(z^{D}\right) \tilde{H}_{i 1}\left(z^{D}\right) \\
=H_{i 0}\left(z^{D}\right) \tilde{H}_{i 0}\left(z^{D}\right)+a_{i} D_{i}(z) z_{1} \tilde{H}_{i 0}\left(z^{D}\right) \\
\quad \cdot a_{i} \tilde{D}_{i}(z) z_{1}^{-1} H_{i 0}\left(z^{D}\right) \\
=2 \cdot H_{i 0}\left(z^{D}\right) \tilde{H}_{i 0}\left(z^{D}\right)=1
\end{aligned}
$$

which is possible only if $H_{i 0}$ and $H_{i 1}$ are constants, i.e., if the filters are two-tap.

\section{Another Visit to the Quincunx Case}

To summarize the important results of this section first note how in two dimensions matrices given in (38) reduce to those for the quincunx lattice introduced in (2). The input/output relation given in (39) for this case reduces to the expression already given in (32). The result from Theorem 4.1 for the quincunx case first appeared in [43]. Since it is going to be used later we state here how the two filters in the analysis bank are related (follows from Corollary 4.2)

$$
\begin{aligned}
\hat{H}_{1}\left(\omega_{1}, \omega_{2}\right)= & -e^{-j\left(\left(k_{1}+k_{2}+1\right) \omega_{1}+\left(k_{1}-k_{2}\right) \omega_{2}\right)} \\
& \cdot \hat{H}_{0}\left(-\omega_{1}+\pi,-\omega_{2}+\pi\right), \\
H_{1}\left(z_{1}, z_{2}\right)= & -z_{1}^{-\left(k_{1}+k_{2}+1\right)} z_{2}^{-\left(k_{1}-k_{2}\right)} \\
& \cdot H_{0}\left(-z_{1}^{-1},-z_{2}^{-1}\right)
\end{aligned}
$$

where the vector $\boldsymbol{k}=\left(k_{1}, k_{2}\right)$ is as stated in Section IV-A large enough to make the entries in the second row of the matrix in (40) causal.

The linear phase case was studied extensively in [43] where similar analysis on possible linear phase solutions was performed but in polyphase domain and for diamond shaped filters. Here we want to see how the Propositions 4.2-4.6 translate for this case. For example, case 1) of Proposition 4.2 would tell us that if $i\left(l_{1}+l_{2}\right)=1$, i.e., if the sizes of the filter in two dimensions are either both odd or both even, then both polyphase components will have the same kind of symmetry as the filter itself has. If, on the other hand, in one dimension filter length is even and in the other one odd, polyphase components do not necessarily have any kind of symmetry but instead one can obtain the second polyphase component by shift-reversing the first one. Next, Proposition 4.4 states that to have a perfect reconstruction pair both filters have either the same symmetry and their sizes are as in case 1) of Proposition 4.2 or they are of opposite symmetry and each is of odd length in one dimension and of even length in the other. The fact that in the two-channel case having linear phase and orthogonal filters is not possible (see Proposition 4.6) is as was said earlier due to the fact that the algebraic structure of the modulation/ polyphase matrices is basically the same regardless of the number of dimensions. Similar reasoning can be used when constructing solutions for the four-channel two-dimensional case that are at the same time orthogonal and have linear phase, namely achieving both at the same time is feasible since it is feasible in the four-channel one-dimensional case as well (see Section V-A-2).

\section{Synthesis of Multidimensional Filter Banks}

\section{A. Cascade Structures}

When synthesizing filter banks one of the most obvious approaches is to try to find cascade structures that would generate filters of the desired form, the reason being that cascade structures: i) usually have very low complexity; ii) higher order filters are easily derived from the lower order ones; and iii) the coefficients can be quantized without affecting the desired form.

While in the orthogonal case forming a cascade that would achieve perfect reconstruction is trivial since one has just to combine orthogonal building blocks (i.e., orthogonal matrices and diagonal delay matrices), in the linear phase case this is not so simple. There one has to make use of the linear phase testing condition given in (23) or [21] to obtain possible cascades. As one of the possible approaches consider the generalization of the linear phase cascade structure proposed in [21], [22], [44]. Suppose that a linear phase system has been already designed and a higher order one is needed. Then choosing

$$
H_{p}^{\prime \prime}(z)=H_{p}^{\prime}(z) \cdot D(z) \cdot R
$$

where $D(z)=z^{-k} \cdot J \tilde{D}(z) J$ and $R$ is persymmetric (i.e., $\boldsymbol{R}=\boldsymbol{J} \boldsymbol{R} \boldsymbol{J})$, another linear phase system is obtained where the filters have the same symmetry as in $\boldsymbol{H}_{p}^{\prime}$. This can be easily verified if substituted into (23). Although this cascade is in no way complete it can produce very useful filters as will be seen later (for examples, refer to Section V-A-1). Let us also point out that while building cascades in the polyphase domain one must bear in mind that using different sampling matrices for the same lattice will greatly affect the geometry of the filters obtained.

1) Cascade Structures for the Two-Channel Case in $n$ Dimensions:

Lemma 5.1: The following cascade will produce a perfect reconstruction set containing two filters of the same size:

$$
\boldsymbol{H}_{p}(z)=\boldsymbol{R}_{0} \cdot \prod_{j=1}^{k} \prod_{i=1}^{n}\left(\begin{array}{cc}
1 & 0 \\
0 & z_{i}^{-1}
\end{array}\right) \boldsymbol{R}_{j_{i}} .
$$

For the filters to be orthogonal the matrices $\boldsymbol{R}_{j_{i}}$ have to be unitary, while for them to be linear phase matrices have to be symmetric. In the latter case the filters obtained will have opposite symmetry. 
Proof: For the orthogonal case it is obvious since all the blocks involved are orthogonal. For the linear phase case use condition (23).

The one-dimensional orthogonal solution obtained by the above cascade is complete (all orthogonal solutions can be reached using it) [35]. The linear phase solution in one dimension generates filters of size $2(k+1)$ and was proposed in [28], [44]. The two-dimensional solution generates filters of sizes $2(k+1) \times(2 k+1)$ and both orthogonal and linear phase filters were proposed in [43]. Note that unlike in the one-dimensional orthogonal case, for linear phase and higher dimensional cascades, completeness results are missing except for very small cases. In [43], it was shown that the smallest solutions both for orthogonal and linear phase cascades are general. The same will be shown in the next lemma, where the smallest size perfect reconstruction filter pairs are complete in any number of dimensions. Moreover, higher dimensional solutions will be generated from lower dimensional ones. In what follows, $z_{u}^{(i)}$ will denote $z^{D}$ where $z=\left(z_{1}, \cdots, z_{i}\right)^{t}$.

Lemma 5.2: 1) The general solution for the perfect reconstruction linear phase set where one filter is of size 3 and the other of size 5 in dimensions $(1, \cdots, n)$ can be generated from a general solution for the perfect reconstruction linear phase set with the same sizes in dimensions $(1, \cdots, n-1)$ with

$$
\begin{aligned}
H_{00}\left(z_{u}^{(n)}\right)= & H_{00}\left(z_{u}^{(n-1)}\right)+a \cdot z_{1}^{-1}\left(z_{n}^{-1}+z_{n}\right), \\
H_{01}\left(z_{u}^{(n)}\right)= & H_{01}\left(z_{u}^{(n-1)}\right), \\
H_{10}\left(z_{u}^{(n)}\right)= & H_{10}\left(z_{u}^{(n-1)}\right)+H_{c}\left(z_{u}^{(n-1)}\right) \cdot z_{1}^{-1}\left(z_{n}^{-1}+z_{n}\right) \\
& +c \cdot z_{1}^{-2}\left(z_{n}^{-2}+z_{n}^{2}\right), \\
H_{11}\left(z_{u}^{(n)}\right)= & H_{11}\left(z_{u}^{(n-1)}\right)+d \cdot z_{1}^{-1}\left(z_{n}^{-1}+z_{n}\right),
\end{aligned}
$$

where

$$
\begin{aligned}
H_{0 \mathrm{t}}\left(z_{u}^{(n-1)}\right) & =b, b \neq 0, \\
H_{c}\left(z_{u}^{(n-1)}\right) & =\frac{d \cdot H_{00}\left(z_{u}^{(n-1)}\right)+a \cdot H_{11}\left(z_{u}^{(n-1)}\right)}{b}, \\
a \cdot d & =b \cdot c, \\
t_{n-1}+2 a d & \neq 0 .
\end{aligned}
$$

Here $t_{n-1}$ is the only nonzero coefficient in the determinant of the polyphase matrix in dimensions $(1, \cdots, n-1)$, i.e., det $H_{p}\left(z_{u}^{(n-1)}\right)=t_{n-1} \cdot z_{1}^{-2}$. The determinant of the polyphase matrix in dimensions $(1, \cdots, n)$ is then $\operatorname{det} \boldsymbol{H}_{p}\left(\boldsymbol{z}_{u}^{(n)}\right)=\left(t_{n-1}+2 a d\right) \cdot z_{1}^{-2}=t_{n} \cdot z_{1}^{-2}$.

2) The cascade of $k$ polyphase matrices as above will generate a linear phase perfect reconstruction filter set of the same shape, where the first filter is of size $(2 k+1)$ and the second one of size $(2 k+3)$ in all dimensions, and where all the polyphase components are $A$-polynomials.

Proof: For proof and properties of $A$-polynomials, see Appendix D.
The one-dimensional solution as in Lemma 5.2 appeared in [44] while a two-dimensional diamond shaped filter pair was proposed in [43]. Basically, the previous lemma gives a possibility to generate $n$-dimensional filters of the above sizes from the $(n-1)$-dimensional ones of the same size. It should be noted that the initial $3 / 5$ solution is completely general regardless of the number of dimensions. The way higher dimensional filters are constructed from the lower dimensional ones is that the lower dimensional solution is kept and then smaller size filters are stacked upon it. An example showing how to construct the two-dimensional (quincunx) solution from the one-dimensional one is given in Section V-C. Note that the cascade obtained in part 2) of the lemma produces a linear phase set where both filters are symmetric. Bearing in mind the fact that they are odd in all dimensions it becomes obvious that they belong to the class 1) solution of Proposition 4.4.

2) How to Generate Cascades Being Orthogonal and Linear Phase: This section will deal with the four-channel separable two-dimensional case; thus the matrix characterizing the sampling process is $D=2 \cdot I$ and the corresponding sampling density is $N=\operatorname{det} D=4$.

Let us first present a cascade structure that will generate four linear phase/orthogonal filters of the same size, where two of them are symmetric and the other two antisymmetric [22]

$$
\boldsymbol{H}_{p}\left(z_{1}, z_{2}\right)=\boldsymbol{W}_{4} \prod_{i=1}^{k} \boldsymbol{D}\left(z_{1}, z_{2}\right) \boldsymbol{R}_{i}
$$

where $W_{4}$ is the matrix representing the Walsh-Hadamard transform of size $4, D$ is the matrix of delays containing the vector of the forward polyphase transform along the diagonal, and $\boldsymbol{R}_{i}$ are scalar persymmetric matrices of the following form:

$$
\boldsymbol{R}_{i}=\left(\begin{array}{cccc}
a_{i 0} & a_{i 2} & a_{i 1} & a_{i 3} \\
a_{i 2} & \pm a_{i 0} & \pm a_{i 3} & a_{i 1} \\
a_{i 1} & \pm a_{i 3} & \pm a_{i 0} & a_{i 2} \\
a_{i 3} & a_{i 1} & a_{i 2} & a_{i 0}
\end{array}\right)
$$

The " - " sign in (47) along with the requirement that the $\boldsymbol{R}_{i}$ be unitary allows one to design filters being both linear phase and orthogonal. In view of the fact that in the two-channel one-dimensional case these two requirements are mutually exclusive (see Proposition 4.6), it becomes obvious that one cannot design separable filters satisfying both properties in this four-channel two-dimensional case. This shows how using a true multidimensional solution offers greater freedom in design. For an example to the previous discussion, refer to [21]. At the same time, if a regular low-pass filter can be found (see Section VI), this cascade would allow one to generate orthogonal bases of linear phase (symmetric) wavelets in two dimensions, a construction which is not possible in current designs based on tensor products of one-dimensional systems. 
The previous cascade generated filters of the same size. Now we show how to generate a cascade structure producing four linear phase filters of sizes $(2 k+3) \times$ $(2 k+3),(2 k+3) \times(2 k+1),(2 k+1) \times(2 k+3)$ and $(2 k+1) \times(2 k+1)$. The basic building block is obtained for $k=1$ and the corresponding filters' impulse responses are shown in Fig. 6. The cascade of such building blocks will produce again a linear phase set of sizes as above and of the same symmetry.

\section{B. One to Multidimensional Transformations}

Because of the difficulty of designing good filters in multiple dimensions, transformations mapping one-dimensional designs into multidimensional ones have been used for some time, the most popular being the McClellan transformation [14], [24].

In the context of filter banks and wavelets, one would like to transform a one-dimensional filter bank into a multidimensional one such that:

1) perfect reconstruction is preserved;

2) zeros at aliasing frequencies are preserved.

The first requirement is obvious, while the second one is necessary to achieve some degree of regularity (see Section VI-B).

Note that iteration of a one-dimensional filter with respect to a nonseparable lattice leads to a multidimensional filter (because upsampling transforms $z$ into $z^{D}$; see (4)). This can be used in order to get multidimensional wavelets, and Cohen and Daubechies [7] have used this techniques to construct smooth wavelets from iterated one-dimensional filters (with respect to the dilation matrix $D$ for the quincunx lattice). However, from a discrete filtering point of view, this is of little interest, since the filters are one-dimensional. In the following, we are going to consider transforms that lead to multidimensional filters.

1) Separable Polyphase Components: A first possible transform is obtained by designing a multidimensional filter having separable polyphase components, given as products of the polyphase components of a one-dimensional filter [1], [6]. To be specific, consider the quincunx subsampling case. Start with a one-dimensional filter having polyphase components $H_{0}(z)$ and $H_{1}(z)$, that is, a filter with a $z$-transform $H(z)=H_{0}\left(z^{2}\right)+z^{-1} H_{1}\left(z^{2}\right)$. Derive separable polyphase components

$$
H_{i}\left(z_{1}, z_{2}\right)=H_{i}\left(z_{1}\right) H_{i}\left(z_{2}\right), \quad i=0,1 .
$$

Then, the two-dimensional filter with respect to the quincunx lattice is given as (by upsampling the polyphase components with respect to $D_{1}$ )

$$
\begin{aligned}
H\left(z_{1}, z_{2}\right)=H_{0}\left(z_{1} z_{2}\right) & H_{0}\left(z_{1} z_{2}^{-1}\right) \\
& +z_{1}^{-1} H_{1}\left(z_{1} z_{2}\right) H_{1}\left(z_{1} z_{2}^{-1}\right) .
\end{aligned}
$$

It can be verified that an $N$ th-order zero at $\pi$ in $H\left(e^{j \omega}\right)$
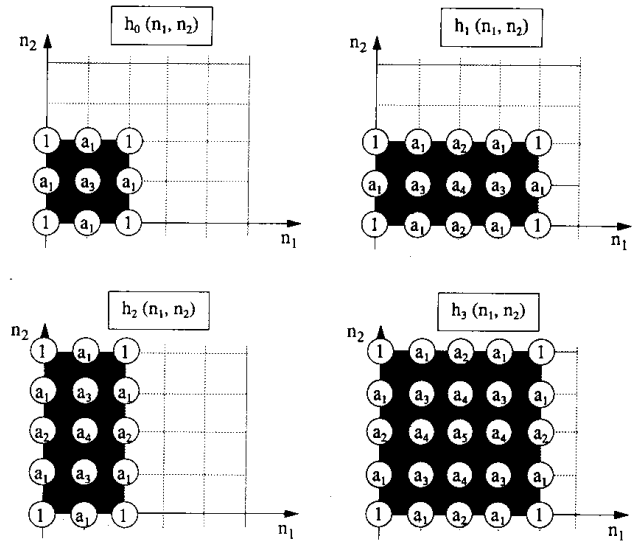

Fig. 6. The impulse responses of the four filters which form the first block in the cascade.

maps into an $N$ th-order zero at $(\pi, \pi)$ for $H\left(e^{j \omega_{1}}, e^{j \omega_{2}}\right)$, and thus property 2) is achieved. However, an orthogonal filter bank is mapped into an orthogonal two-dimensional bank, if and only if the polyphase components of the onedimensional filter are all-pass functions (that is, $H_{i}\left(e^{j \omega}\right) H_{i}\left(e^{-j \omega}\right)=c$, see $\left.(16)\right)$. Perfect reconstruction is thus not conserved in general. Note that the separable polyphase components lead to efficient implementations, reducing the number of operations from $O\left[l^{2}\right]$ to $O[l]$ per output where $l$ is the filter size.

2) McClellan Transformation: The Fourier transform of a zero phase symmetric filter $(h(n)=h(-n))$ can be written as a function of $\cos (n \omega)$

$$
\hat{H}(\omega)=\sum_{n=0}^{L} a(n) \cos (n \omega)
$$

where $a(0)=h(0)$ and $a(n)=2 h(n), \quad n \neq 0 . \quad$ Using Tchebycheff polynomials, one can replace $\cos (n \omega)$ by $T_{n}[\cos (\omega)]$ where $T_{n}[\cdot]$ is the $n$th Tchebycheff polynomial, and thus $\hat{H}(\omega)$ can be written as a polynomial of $\cos (\omega)$

$$
\hat{H}(\omega)=\sum_{n=0}^{L} a(n) T_{n}[\cos (\omega)]
$$

The idea of the McClellan transformation is to replace $\cos (\omega)$ by a zero phase two-dimensional filter $\hat{F}\left(\omega_{1}, \omega_{2}\right)$, hence resulting in an overall zero phase two-dimensional filter [14], [24]

$$
\hat{H}(\omega)=\sum_{n=0}^{L} a(n) T_{n}\left[\hat{F}\left(\omega_{1}, \omega_{2}\right)\right]
$$

In the context of filter banks, this transformation can only be applied to the biorthogonal case (because of the zero phase requirement). Typically, in the case of quincunx subsampling, $\hat{F}\left(\omega_{1}, \omega_{2}\right)$ is chosen as [3], [7]

$$
\hat{F}\left(\omega_{1}, \omega_{2}\right)=\frac{1}{2}\left(\cos \left(\omega_{1}\right)+\cos \left(\omega_{2}\right)\right) .
$$


That the perfect reconstruction is preserved can be checked by considering the determinant of the matrix $\hat{H}_{m}(\omega)$ in (15) which is a monomial in the one-dimensional case since one starts with a perfect reconstruction filter bank. The transformation in (53) leads to a determinant of $\hat{H}_{m}\left(\omega_{1}, \omega_{2}\right)$ which is also a monomial, and thus, perfect reconstruction is conserved.

In addition to this, it is easy to see that pairs of zeros at $\pi$ (that is, factors of the form $1+\cos (\omega)$ ) map into zeros of order two at $(\pi, \pi)$ in the transformed domain (or factors of the form $\left.1+1 / 2 \cos \left(\omega_{1}\right)+1 / 2 \cos \left(\omega_{2}\right)\right)$.

Therefore, the McClellan transform is a powerful method to map one-dimensional biorthogonal solutions to multidimensional biorthogonal solutions, and this while conserving zeros at aliasing frequencies.

\section{Cascade Structure for the Quincunx Case}

As an illustration to Section V-A-1, the $3 / 5$ set from Lemma 5.2 for the two-dimensional (quincunx) case will be constructed. That solution, in turn, is used in [19] for constructing filters for the FCO case (three-dimensional nonseparable two-channel case). We start from the onedimensional solution for a general polyphase matrix in the upsampled domain [44] $H_{00}\left(z_{1}^{2}\right)=1+z_{1}^{-2}, H_{01}\left(z_{1}^{2}\right)=a_{1}$, $H_{10}\left(z_{1}^{2}\right)=1+a_{2} z_{1}^{-2}+z_{1}^{-4}, H_{11}\left(z_{1}^{2}\right)=a_{1}\left(1+z_{1}^{-2}\right)$. To construct the two-dimensional solution one needs to evaluate the polynomial

$$
\begin{aligned}
H_{c}\left(z_{1}^{2}\right) & =\frac{d \cdot\left(1+z_{1}^{-2}\right)+a \cdot a_{1}\left(1+z_{1}^{-2}\right)}{a_{1}} \\
& =\left(a+\frac{d}{a_{1}}\right)\left(1+z_{1}^{-2}\right)
\end{aligned}
$$

and also to express one of the variables $a, c$, and $d$ using the two other ones. Thus, writing $c=a d / a_{1}$ the following is obtained (note that $\left(z_{1}, z_{2}\right)$ in the upsampled domain is $\left(z_{1} z_{2}, z_{1} z_{2}^{-1}\right)$ as given in (8))

$$
\begin{aligned}
& H_{00}\left(z_{1} z_{2}, z_{1} z_{2}^{-1}\right)= 1+z_{1}^{-2}+a \cdot z_{1}^{-1}\left(z_{2}^{-1}+z_{2}\right) \\
& H_{01}\left(z_{1} z_{2}, z_{1} z_{2}^{-1}\right)= a_{1}, \\
& H_{10}\left(z_{1} z_{2}, z_{1} z_{2}^{-1}\right)=1+a_{2} z_{1}^{-2}+z_{1}^{-4}+\left(a+\frac{d}{a_{1}}\right) \\
& \cdot\left(1+z_{1}^{-2}\right) z_{1}^{-1}\left(z_{2}^{-1}+z_{2}\right) \\
&+\frac{a d}{a_{1}} \cdot z_{1}^{-2}\left(z_{2}^{-2}+z_{2}^{2}\right), \\
& H_{11}\left(z_{1} z_{2}, z_{1} z_{2}^{-1}\right)= a_{1}\left(1+z_{1}^{-2}\right)+d \cdot z_{1}^{-1}\left(z_{2}^{-1}+z_{2}\right)
\end{aligned}
$$

which yields the following impulse responses of the filters:

$$
\begin{aligned}
& h_{0}\left(n_{1}, n_{2}\right)=\left(\begin{array}{ccc} 
& a & \\
1 & a_{1} & 1 \\
& a &
\end{array}\right)
\end{aligned}
$$

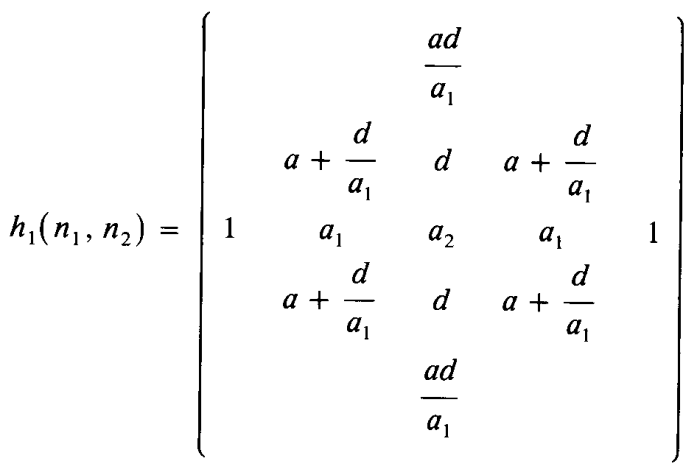

which is the same solution as obtained in [43]. Using the result of Lemma 5.2 one can see that the cascades of the building blocks from (55) would generate diamond shaped filters of sizes $(2 k+1) \times(2 k+1)$ and $(2 k+3) \times$ $(2 k+3)$ conserving the perfect reconstruction property. Fig. 7 shows how the smallest size filters are obtained. Note that if one would give up some freedom in design and impose additional circular symmetry, this solution would become the same as the one obtained by using McClellan transformation as explained in Section V-B-2. As will be seen in Section VII, this example will allow us to obtain a filter bank where the analysis low-pass can be made highly regular.

\section{Connection to Nonseparable Wavelet Bases}

\section{A. Constructing Wavelet Bases from Iterated Filter Banks}

Let us first recall the one-dimensional case. There it is obvious that an orthogonal perfect reconstruction filter bank computes the discrete-time wavelet transform when the branch with the low-pass filter is iterated. Also, under certain conditions the same filter bank can be used to obtain a continuoustime wavelet transform [9], [23], [41], [42]. In multiple dimensions the basic ideas are the same, the only difference being that instead of dealing with dilation factors we deal with a dilation matrix.

Thus, consider Fig. 8. The equivalent low branch after $i$ steps of filtering and sampling by $\boldsymbol{D}$ will contain the following filter and sampling by $\boldsymbol{D}^{i}$ :

$$
\hat{H}^{(i)}(\omega)=\prod_{k=0}^{i-1} \hat{H}_{0}\left(\left(D^{t}\right)^{i} \omega\right) \quad i=1,2, \cdots
$$

where $\hat{H}^{(0)}(\omega)=1$ and the fact that sampling by $D$ followed by filtering by $\hat{H}(\omega)$ is equivalent to filtering by $\hat{H}\left(D^{t} \omega\right)$ followed by sampling by $D$ was used. The aim is to construct a continuous-time function corresponding to $h^{(i)}(n)$, the latter being the impulse response of the iterated filter $\hat{H}^{(i)}(\omega)$. Consequently, we define

$$
f^{(i)}(x)=N^{i / 2} \cdot h^{(i)}(n) \quad D^{i} x \in n+\left[-\frac{1}{2}, \frac{1}{2}\right)^{n} .
$$

Obviously, $f^{(0)}(x)$ is just the indicator function over the 

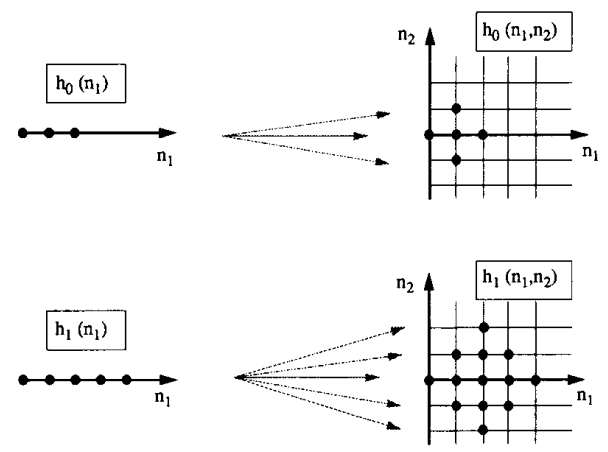

Fig. 7. The process of obtaining two-dimensional linear phase set from the one-dimensional one.

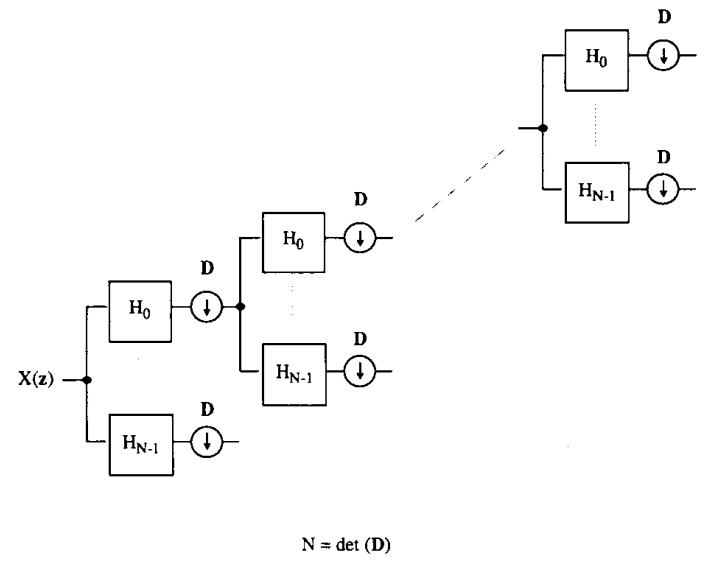

Fig. 8. Computation of the discrete wavelet transform using a filter bank.

hypercube $\left[-\frac{1}{2}, \frac{1}{2}\right)^{n}$. The function in (57) is constant over regions of hypervolume $1 / N^{i}$ and normalization by $N^{i / 2}$ is chosen so that if $\left\|h^{(i)}(n)\right\|_{2}=1$, then $\left\|f^{(i)}(x)\right\|_{2}=1$ as well. Note that the shape of the hyperregions defined in (57) is not rectangular, rather it is determined by the shape of the unit cell belonging to the lattice $D^{-i}$. As in the onedimensional case we are interested in the limiting behavior of the iterated function. Thus, we conjecture that the iterated filter can be made regular (i.e., the iterated function continuous) by putting a sufficient number of zeros at the points in frequency domain where the repeated spectra occur, i.e., at the points belonging to the reciprocal lattice of $D$ (see the discussion in Section II).

In what follows, except for the necessity of having a zero at aliasing frequencies (see Section VI-B), we will not deal with the problem of achieving the continuous limit of $f^{(i)}(\boldsymbol{x})$. This is discussed in detail in [7] for the quincunx case. We will instead start by assuming that the limit of $f^{(i)}(\boldsymbol{x})$ exists and is in $L^{2}$ and will call it the scaling function associated with the discrete filter $h_{0}(\boldsymbol{n})$

$$
\phi(x)=\lim _{i \rightarrow \infty} f^{(i)}(x), \quad \phi(x) \in L^{2}
$$

Morever, we will assume for simplicity, that this limit function is continuous. We want to show now that the scaling function satisfies the so-called two-scale equation [9], [11], [32], [41]. Following (56) we can write the equivalent filter after $i$ steps in terms of the equivalent filter after $(i-1)$ steps as

$$
h^{(i)}(n)=\sum_{k} h_{0}(\boldsymbol{k}) h^{(i-1)}\left(n-D^{i-1} k\right) .
$$

Using (57) we want to express the previous equation in terms of iterated functions and thus

$$
\begin{aligned}
h^{(i)}(\boldsymbol{n}) & =N^{-i / 2} \cdot f^{(i)}(\boldsymbol{x}), \\
h^{(i-1)}\left(\boldsymbol{n}-\boldsymbol{D}^{i-1} \boldsymbol{k}\right) & =N^{-(i-1) / 2} \cdot f^{(i-1)}(\boldsymbol{D} \boldsymbol{x}-\boldsymbol{k})
\end{aligned}
$$

both for $\boldsymbol{D}^{i} \boldsymbol{x} \in \boldsymbol{n}+\left[-\frac{1}{2}, \frac{1}{2}\right)^{n}$. Substituting equations (60) and (61) into (59) yields

$$
f^{(i)}(\boldsymbol{x})=\sqrt{N} \sum_{\boldsymbol{k}} h_{0}(\boldsymbol{k}) f^{(i-1)}(\boldsymbol{D} \boldsymbol{x}-\boldsymbol{k}) .
$$

Recall that the assumption is that the iterated function $f^{(i)}(\boldsymbol{x})$ converges to the scaling function. Hence, we can take the limits of both sides of $(62)$ to obtain

$$
\phi(x)=\sqrt{N} \sum_{\boldsymbol{k}} h_{0}(\boldsymbol{k}) \phi(\boldsymbol{D} \boldsymbol{x}-\boldsymbol{k})
$$

showing that indeed the scaling function satisfies the two-scale equation. Note that in the multidimensional case the change of scale involves the dilation matrix $D$. Until now we have been concerned only with the iterated low-pass filter. But what happens with other branches in the last stage of iteration? Following the same arguments as before, each one of them can be expressed as the iterated low-pass filter $h^{(j)}(n)$ followed by one filter $h_{i}(n)$. Since the former one tends to $\phi(\boldsymbol{x}),(N-1)$ wavelets can be defined, each one satisfying

$$
\begin{aligned}
\psi_{i}(\boldsymbol{x}) & =\sqrt{N} \sum_{\boldsymbol{k}} h_{i}(\boldsymbol{k}) \boldsymbol{\phi}(\boldsymbol{D} \boldsymbol{x}-\boldsymbol{k}) \\
i & =1,2, \cdots, N-1 .
\end{aligned}
$$

Refer to Section III-B. There the orthogonality relations between filters and their translates with respect to the sampling lattice were shown (see (21)). We want to use those results to obtain the same kind of relationships for the scaling function and the $(N-1)$ wavelets. Here, we just state the facts and outline the proof for the first one, the others would follow similarly:

1) $\langle\phi(x), \phi(x-l)\rangle=\delta_{l}$, that is, the scaling function is orthogonal to its integer translates;

2) $\left\langle\phi\left(D^{i} x-l\right), \phi\left(D^{i} x-k\right)\right\rangle,=N^{-i} \delta_{l k}$, i.e., the previous fact holds for all scales;

3) $\left\langle\psi_{m}\left(\boldsymbol{D}^{i} \boldsymbol{x}-\boldsymbol{l}\right), \psi_{n}\left(\boldsymbol{D}^{i} \boldsymbol{x}-\boldsymbol{k}\right)\right\rangle=N^{-i} \delta_{m n} \delta_{l k}$, wavelets are orthogonal to each other and their integer translates;

4) $\left\langle\phi(x), \psi_{m}(x-l)\right\rangle=0$, the scaling function is orthogonal to each of the wavelets; and

5) $\left\langle\psi_{m}\left(\boldsymbol{D}^{i} \boldsymbol{x}-\boldsymbol{l}\right), \psi_{n}\left(\boldsymbol{D}^{j} \boldsymbol{x}-\boldsymbol{k}\right)\right\rangle=N^{-i} \delta_{m n} \delta_{i j} \delta_{l \boldsymbol{k}}$, wavelets are orthogonal across scales. 
To prove the first fact we use induction on the function $f^{(i)}$ and then take the limit (which exists by assumption). The first step $\left\langle f^{(0)}(\boldsymbol{x}), f^{(0)}(\boldsymbol{x}-\boldsymbol{k})\right\rangle=\delta_{\boldsymbol{k}}$ is obvious since by definition $f^{(0)}(\boldsymbol{x})$ is just the indicator function on the hypercube $x \in\left[-\frac{1}{2}, \frac{1}{2}\right)^{n}$. For the inductive step we can write

$$
\begin{aligned}
\left\langle f^{(i+1)}(\boldsymbol{x}), f^{(i+1)}(\boldsymbol{x}-\boldsymbol{l})\right\rangle & \\
= & \left(\sqrt{N} \sum_{\boldsymbol{k}} h_{0}(\boldsymbol{k}) f^{(i)}(\boldsymbol{D} \boldsymbol{x}-\boldsymbol{k}),\right. \\
& \left.\sqrt{N} \sum_{\boldsymbol{m}} h_{0}(\boldsymbol{m}) f^{(i)}(\boldsymbol{D} \boldsymbol{x}-\boldsymbol{D} \boldsymbol{l}-\boldsymbol{m})\right), \\
= & N \sum_{\boldsymbol{k}} \sum_{\boldsymbol{m}} h_{0}(\boldsymbol{k}) h_{0}(\boldsymbol{m}) \\
& \cdot\left\langle f^{(i)}(\boldsymbol{D} \boldsymbol{x}-\boldsymbol{k}), f^{(i)}(\boldsymbol{D} \boldsymbol{x}-\boldsymbol{D} \boldsymbol{l}-\boldsymbol{m})\right\rangle, \\
= & \sum_{\boldsymbol{m}} h_{0}(\boldsymbol{m}) h_{0}(\boldsymbol{D} \boldsymbol{l}+\boldsymbol{m}), \\
= & \left\langle h_{0}(\boldsymbol{m}), h_{0}(\boldsymbol{D} \boldsymbol{l}+\boldsymbol{m})\right\rangle=\delta_{\boldsymbol{l}}
\end{aligned}
$$

where we used (21). Taking the limit of both sides of the previous equation we get exactly the first fact.

We have thus verified that

$$
\begin{array}{r}
S=\left\{N^{-m / 2} \psi_{i}\left(\boldsymbol{D}^{-m} \boldsymbol{x}-\boldsymbol{n}\right) \mid i=\right. \\
\qquad, \cdots, N-1, m \in \mathscr{Z}, \\
\left.n \in \mathscr{Z}^{n}, \boldsymbol{x} \in \mathscr{R}^{n}\right\}
\end{array}
$$

is an orthonormal set. The only thing left to do is to show that the members of the set $S$ constitute an orthonormal basis for $L^{2}\left(\mathscr{R}^{n}\right)$. The way to do it is to verify that $S$ is a tight frame with framebound equal to one [9]. In Section VI-D we will show that this is indeed true for the two-channel case in any number of dimensions.

\section{B. Necessity of Zeros at Aliasing Frequencies}

In this section we want to extend Rioul's one-dimensional result on the necessity of a zero at $\pi$ [29]. This result then makes it plausible why one would try to impose a zero of a sufficiently high order at aliasing frequencies (or points of repeated spectra).

Theorem 6.1: If the scaling function $\phi(x)$ exists for some $x \in \mathscr{R}^{n}$, then

$$
\sum_{\boldsymbol{k} \in \mathscr{Y}^{n}} h\left(D \boldsymbol{k}+\boldsymbol{k}_{i}\right)=\frac{1}{\sqrt{N}}, \quad \boldsymbol{k}_{i} \in \mathscr{U}_{c},
$$

or in other words

$$
\begin{aligned}
\hat{H}(\boldsymbol{\omega}=\mathbf{0})=\sqrt{N}, \quad \hat{H}\left(\boldsymbol{\omega}=2 \pi\left(D^{t}\right)^{-1} \boldsymbol{n}\right) & =0, \\
n & \in \mathscr{Z}^{n}
\end{aligned}
$$

where $2 \pi\left(D^{\prime}\right)^{-1} n$ are the aliasing frequencies or the points of repeated spectra.

Proof: Here we give just an outline of the proof; for more details refer to [18]. Note first that we can write (59) as

$$
h^{(i)}(\boldsymbol{n})=\sum_{\boldsymbol{k}} h^{(i-1)}(\boldsymbol{k}) h_{0}(\boldsymbol{n}-\mathrm{Dk})
$$

and thus

$$
h^{(i)}(D n)=\sum_{k} h_{0}(D k) h^{(i-1)}(n-k) .
$$

Using the same approach as in derivation (60)-(63), express $h^{(i-1)}$ and $h^{(i)}$ in terms of $f^{(i-1)}$ and $f^{(i)}$ and then take the limits (we are allowed to do so by assumption)

$$
\phi(D \boldsymbol{x})=\sqrt{N} \sum_{\boldsymbol{k}} h_{0}(\boldsymbol{D} \boldsymbol{k}) \phi(\boldsymbol{D} \boldsymbol{x}) .
$$

Writing (69) for all the elements of the unit cell, i.e., $h^{(i)}\left(D n+n_{i}\right), n_{i} \in \mathscr{Z}_{c}$, and following the same path as for $h^{(i)}(D n)$ we finally obtain

$$
\phi(D x)=\sqrt{N} \sum_{k} h_{0}\left(\boldsymbol{D} \boldsymbol{k}+\boldsymbol{k}_{i}\right) \phi(\boldsymbol{D} \boldsymbol{x}), \quad \forall \boldsymbol{k}_{i} \in \mathscr{H}_{c} .
$$

Equating (71) for all the various coset representatives results in (66).

\section{Choice of the Dilation Matrix}

In the Introduction we mentioned that the dilation matrix $D$ must satisfy the following conditions:

1) $\left|\lambda_{i}\right|>1, \forall i$, and

2) $D \mathscr{Z}^{n} \subseteq \mathscr{Z}^{n}$

where $\lambda_{i}$ denote the eigenvalues of the matrix $D$. The first condition ensures that there is indeed a dilation in each dimension [7], [16]. Such a matrix will be called a wellbehaved matrix. From the discrete filtering point of view, we would also like to use matrices that lead to separable sampling after a small number of iterations. That is the reason why we use the matrix $D_{1}$ rather than $D_{2}$ when dealing with the quincunx case (see Sections VI-E and VII). By the same token we would use the following matrices for the hexagonal and FCO lattices

$$
\begin{array}{lll}
\boldsymbol{D}_{H E X}=\left(\begin{array}{rr}
2 & 1 \\
0 & -2
\end{array}\right), & D_{H E X}^{2}=\left(\begin{array}{ll}
4 & 0 \\
0 & 4
\end{array}\right), \\
\boldsymbol{D}_{F C O}=\left(\begin{array}{rrr}
1 & 0 & 1 \\
-1 & -1 & 1 \\
0 & -1 & 0
\end{array}\right), & \boldsymbol{D}_{F C O}^{3}=\left(\begin{array}{lll}
2 & 0 & 0 \\
0 & 2 & 0 \\
0 & 0 & 2
\end{array}\right) .
\end{array}
$$

At the same time, the examples discovered by Gröchenig and Madych in [16], as well as Lawton and Resnikoff [49], that use equivalents of Haar bases for filters and by construction their iterates are self-similar and tile the space, show that vastly different behavior is obtained when using different matrices for the same lattice. For example, the matrix $\boldsymbol{D}_{2}$ ("twin dragon" in the Haar case) would lead to fractal support while $D_{1}$ would lead to parallelepiped support.

Fig. 9 gives an interesting example of a "dragon" for the hexagonal lattice. It has been generated using the lattice given in (72) with the following filter $H\left(z_{1}, z_{2}\right)=1+$ $z_{1}^{-1}+z_{1}^{-1} z_{2}^{-1}+z_{1}^{-1} z_{2}$. The plot gives the sixth iteration (plotted on the rectangular grid since the sixth iteration corresponds to separable sampling). When the unit cell of this lattice is used as a basic filter, one obtains the patterns similar to those in [16] with the dilation matrix $\left(\begin{array}{ll}2 & 1 \\ 0 & 2\end{array}\right)$. 


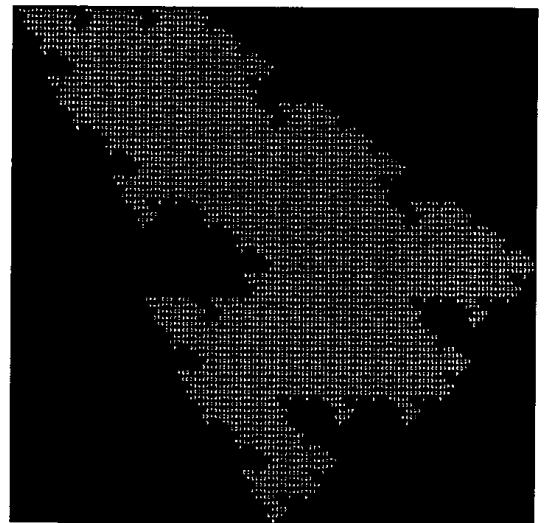

Fig. 9. A dragon obtained when iterating $H\left(z_{1}, z_{2}\right)=1+z_{1}^{-1}+$ $z_{1}^{-1} z_{2}^{-1}+z_{1}^{-1} z_{2}$ with the dilation matrix as in (72). Sixth iteration is given plotted on the rectangular grid.

\section{Two-Channel Orthonormal Bases}

Assume that we are dealing with the $n$-dimensional twochannel case characterized by a matrix $\boldsymbol{D}$, with $\mid$ det $\boldsymbol{D} \mid=2$ and $D$ is a well-behaved matrix (see Section VI-C). Suppose now the following for $h_{0}(\boldsymbol{n}), h_{1}(\boldsymbol{n}), \phi(\boldsymbol{x})$, and $\psi(\boldsymbol{x})$ where $n$ and $\boldsymbol{x}$ are $n$-dimensional integer and real vectors, respectively.

If:

1) filters $h_{0}$ and $h_{1}$ are orthogonal to each other and their translates (as given in (21));

2) the low-pass filter has a zero at aliasing frequencies (see $(67)$ );

3) the filters are FIR;

4) $h_{1}$ is specified from $h_{0}$ by the statement of Corollary 4.2 ;

5) the scaling function is the limit of the iterated functions as given by $(58)$;

6) the wavelet is a linear combination of the scaling function and its shifts (see (64)); and

7) the scaling function and the wavelet are orthogonal to each other and their integer translates across scales (see the enumerated properties that appear after (64));

then (note that 4) follows from 1) and 7) follows from 5)).

Theorem 6.2: The orthonormal set of functions $S=$ $\left\{\psi_{m} n \mid m \in \mathscr{Z}, \boldsymbol{n} \in \mathscr{Z}^{n}, \boldsymbol{x} \in \mathscr{R}^{n}\right\} \quad$ where $\psi_{m n}=2^{-m / 2}$ $\cdot \psi\left(D^{-m} \boldsymbol{x}-\boldsymbol{n}\right)$ is a basis for $L^{2}\left(\mathscr{R}^{n}\right)$, i.e., for $\forall f \in L^{2}\left(\mathscr{R}^{n}\right)$

$$
\sum_{m \in \not{Z} \in \mathscr{Z}^{n}}\left|\left\langle\psi_{m n}, f\right\rangle\right|^{2}=\|f\|_{2}^{2}
$$

Proof: The proof of the theorem is the $n$-dimensional version of the proof given in [9] with appropriate modifications pertaining to $n$-dimensional Fourier transform, and with the dilation matrix $D$ instead of the dilation factor 2 . For more details, we refer the reader to [18].

\section{E. Final Visit to the Quincunx Case}

For the purpose of the following analysis we will use the matrix $D_{1}$ as given by (2) in the Introduction, the reason being that when iterated this matrix would lead to separable sampling in every other step. Thus, this gives us an opportunity to check all of our results in the case that is very well understood, namely two dimensions with dilation factors of 2 in each one of them. As noted earlier, the quincunx case corresponds to a two-channel filter bank. Consequently, we will have a scaling function and one wavelet. To be consistent with our previous notation the low-pass filter is denoted by $h_{0}$ and the high-pass by $h_{1}$.

Let us first see how the "graphical" function defined in (57) looks like

$$
\begin{aligned}
& f^{(i)}\left(x_{1}, x_{2}\right)=2^{i / 2} \cdot h_{0}^{(i)}\left(n_{1}, n_{2}\right) \\
& \quad\left(\begin{array}{rr}
1 & 1 \\
1 & -1
\end{array}\right)^{i}\left(\begin{array}{l}
x_{1} \\
x_{2}
\end{array}\right) \in\left(\begin{array}{l}
n_{1} \\
n_{2}
\end{array}\right)+\left[-\frac{1}{2}, \frac{1}{2}\right) \times\left[-\frac{1}{2}, \frac{1}{2}\right) .
\end{aligned}
$$

As we said, the regions as defined above are not in general rectangular. To see that, consider what happens for $\left(n_{1}, n_{2}\right)=(0,0)$ and the first few iterations. Fig. 10 shows these regions for $i=1,2,3$. For $i=0$ the basic support is just the square $\left[-\frac{1}{2}, \frac{1}{2}\right) \times\left[-\frac{1}{2}, \frac{1}{2}\right)$ as can be seen from (75). In the first and the third iteration the regions are diamonds (tilted squares), while in the second one it is a square as we expected because that case (second iteration) corresponds to separable sampling. We conjecture that to make this graphical function continuous we have to place a zero of a sufficiently high order at the points of repeated spectra, i.e., at $(\pi, \pi)$ (following the discussion in Section VI-B we know that at least one zero is necessary). For a design example using this criterion see the next section and Fig. 11 where the tenth iteration is given (the regions over which the function is plotted are square). The limit function as obtained in (63)

$$
\phi(x)=\sqrt{2} \sum_{k} h_{0}(k) \phi\left(D_{1} x-k\right)
$$

thus satisfies a two-scale equation with respect to scale change given by $D_{1}$ and is orthogonal to its integer shifts. Similarly, the wavelet obtained in (64)

$$
\psi(x)=\sqrt{2} \sum_{k} h_{1}(k) \phi\left(D_{1} x-k\right)
$$

together with its integer shifts and scales by $D_{1}$ will form an orthonormal set.

\section{Design of Compactly Supported Wavelets}

The design of multidimensional filters is a difficult task from a signal processing point of view, but it becomes all the more involved by introducing the requirement that the lowpass filter be regular. To design a filter having a number of vanishing moments at a particular location in one dimension is made possible by the existence of the factorization theorem. Following our conjecture, we would like to do the same 


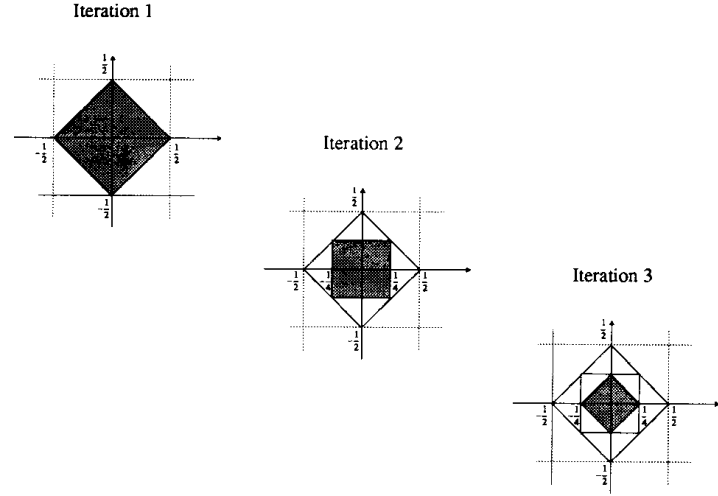

Fig. 10. Shaded regions show the basic supports obtained when iterating the continuous function as given in (75) for the quincunx case.

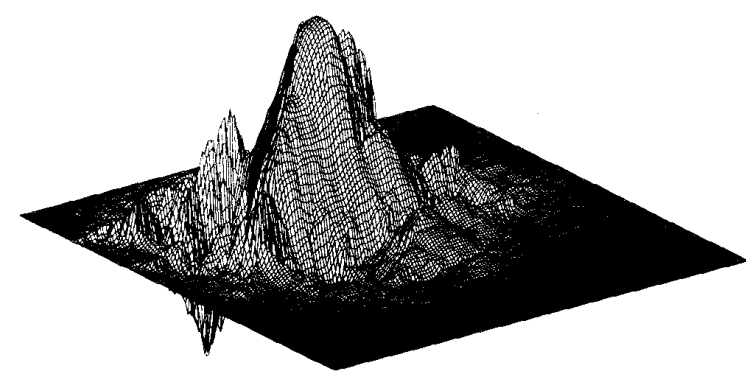

Fig. 11. Tenth iteration of a filter in (78) with coefficients as in (79). The dilation matrix used is $D_{1}$ from (2). The plot is given on the rectangular grid.

for the multidimensional case, but unfortunately factorization theorems are lacking. Thus solving the problem, except for very small cases, has to be done numerically. If one wants an orthogonal solution the equivalent system of equations becomes nonlinear. Therefore, here we show a very small orthogonal design example that was obtained algebraically solving a system of nonlinear equations. First note that the filter bank is obtained using the cascade structure from Lemma 5.1 where matrices $\boldsymbol{R}_{j_{i}}$ are unitary and the sampling matrix used is again $D_{1}$ from (2). Here we use the smallest $(4 \times 3)$ filer pair, since it was shown in [43], that it is general and thus we know that the search space is complete. The impulse response of the low-pass filter is

$$
h_{0}\left(n_{1}, n_{2}\right)=\left(\begin{array}{cccc} 
& -a_{1} & -a_{0} a_{1} & \\
-a_{2} & -a_{0} a_{2} & -a_{0} & 1 \\
& a_{0} a_{1} a_{2} & -a_{1} a_{2} &
\end{array}\right) \text {. }
$$

Following our conjecture (see Section VI) the approach would be to try to impose as high as possible an order of a zero at $(\pi, \pi)$. An $m$ th-order zero means that all the partial derivatives $\left.\left(\partial^{k-1} H_{\omega}\left(\omega_{1}, \omega_{2}\right) / \partial^{l} \omega_{1} \partial^{k-l-1} \omega_{2}\right)\right|_{(\pi, \pi)}=0, \quad k=$ $1, \cdots, m-1, l=0, \cdots, k-1$. Upon imposing a secondorder zero, the following solutions are obtained:

$$
\begin{array}{lll}
a_{0}=\mp \sqrt{3} & a_{1}=\mp \sqrt{3} & a_{2}=2 \pm \sqrt{3}, \\
a_{0}= \pm \sqrt{3} & a_{1}=0 & a_{2}=2 \pm \sqrt{3} .
\end{array}
$$

The solution obtained in $(80)$ is the one-dimensional Daubechies' D4 filter [9]. Hence, we conjecture that the first solution (actually there are two of them but they are related by reversal) would be the smallest "regular" two-dimensional filter. Fig. 11 shows the tenth iteration of the filter in (78) with coefficients as in (79) plotted using matrix $D_{1}$ from (2). The plot is given on the rectangular grid. As can be seen from the figure the obtained function looks continuous although not differentiable at some points. As one simple check of continuity we computed the largest first-order differences in the first seven iterations on the rectangular (or 14 on the quincunx) grid. They are given in Table I. As can be seen from the table the largest difference decreases with an almost constant rate which is a good indicator of a function being continuous. For the purpose of this calculation the filter was normalized so that its $l^{2}$ norm is 1 , and the differences were computed for the iterated function as given in (75). Using the result of the Theorem 6.2 one can then conjecture that the above filter would lead to an orthonormal basis of a compactly supported wavelet. Let us note that Daubechies and Cohen discovered with the same example [7].

For larger size filters problems start to arise. First, the cascade structure given by Lemma 5.1 not being complete we are not even sure we are searching over the whole space of possible solutions. Next, even for the first larger size, namely a filter of size $(6 \times 5)$, the system of nonlinear equations could not be solved analytically. Thus, already for this case having five free variables one would have to resort to numerical solutions.

Turning to the linear phase case, we used the cascade structure given by Lemma 5.2 , the reason being that the diamond-shaped structure allows us to have a highly regular low-pass obtained when convolving the filter $h_{0}\left(n_{1}, n_{2}\right)$ from (55) (with $a=1$ and $a_{1}=4$ )

$$
h_{0}\left(n_{1}, n_{2}\right)=\left(\begin{array}{rrr} 
& 1 & \\
1 & 4 & 1 \\
& 1 &
\end{array}\right)
$$

a number of times with itself. Writing the Fourier-domain expression for (81) one obtains

$$
\begin{aligned}
\hat{H}_{0}\left(\omega_{1}, \omega_{2}\right)=1+e^{-j 2 \omega_{1}} & +e^{-j\left(\omega_{1}+\omega_{2}\right)} \\
& +e^{-j\left(\omega_{1}-\omega_{2}\right)}+4 e^{-j \omega_{1}}
\end{aligned}
$$

showing that it possesses a second-order zero at $(\pi, \pi)$. Although not differentiable, it is continuous [12] (see Fig. 12). However, since the filter bank is not orthogonal, the synthesis low-pass is not equal to the analysis low-pass, but rather to the analysis high-pass modulated by $(-1)^{n_{1}+n_{2}}$. Thus, taking the synthesis low-pass from the cascade given in Lemma 5.2 we imposed a second-order zero at $(\pi, \pi)$ along with some additional symmetry resulting in the following impulse response:

$$
g_{0}\left(n_{1}, n_{2}\right)=\left(\begin{array}{rrrrr} 
& & 1 & & \\
& 2 & -4 & 2 & \\
1 & -4 & -28 & -4 & 1 \\
& 2 & -4 & 2 &
\end{array}\right)
$$




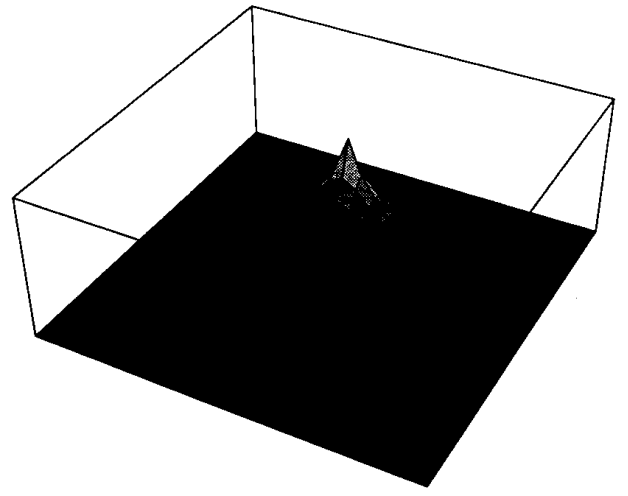

Fig. 12. Sixth iteration of the filter in (81). The dilation matrix used is $D_{1}$ from (2). The plot is given on the rectangular grid.

TABLE I

The Successive Largest First-Order Differences for the Filter Given in (78) with Coefficients as in (79) Computed on the Rectangular Grid

\begin{tabular}{ccc}
\hline $\begin{array}{c}\text { Iteration } \\
\text { Number }\end{array}$ & $\begin{array}{c}\text { Largest first } \\
\text { Order Difference }\end{array}$ & $\begin{array}{c}\text { Rate of } \\
\text { Convergence }\end{array}$ \\
\hline 2 & 1.25163960 & \\
4 & 0.91034730 & 1.3749 \\
6 & 0.62581208 & 1.4547 \\
8 & 0.55111247 & 1.1355 \\
10 & 0.51048814 & 1.0796 \\
12 & 0.46069373 & 1.1081 \\
14 & 0.40993778 & 1.1238 \\
\hline
\end{tabular}

Unfortunately, it turns out that the above filter leads to a fractal iterated function (see Fig. 13(a) and (b)). Daubechies and Cohen in [7] show that a regular synthesis low-pass corresponding to the analysis low-pass as given in (81) would be a diamond of size 57, that is, impractical.

As we mentioned earlier, the filter given in (81) allows one to obtain filters with arbitrarily high regularity. When convolved just once $\left(\hat{H}_{0}^{2}\left(\omega_{1}, \omega_{2}\right)\right)$ it already yields a continuous and differentiable iterated filter [7] as can be seen from Fig. 14 (sixth iteration is shown).

\section{CONCLUSION}

This paper presented new results on multidimensional filter banks and their connection to multidimensional nonseparable wavelets.

Many results are similar to their one-dimensional counterparts, but with the dilation factor replaced by a dilation matrix $\boldsymbol{D}$. This matrix plays a central role: its nonuniqueness for a given lattice becomes important because iterated filter banks (which are the key to our construction of wavelets following [9]) lead to taking powers of $\boldsymbol{D}$. Thus, very unlike the one-dimensional case, a given filter can lead to vastly different scaling functions depending on $\boldsymbol{D}$.

Design techniques for filters leading to regular wavelets do not carry over to the multidimensional case easily, as expected. Therefore, the design of regular wavelets in multi-

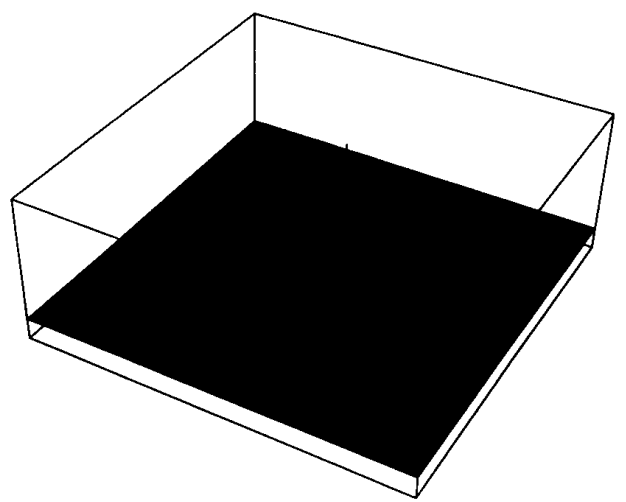

(a)

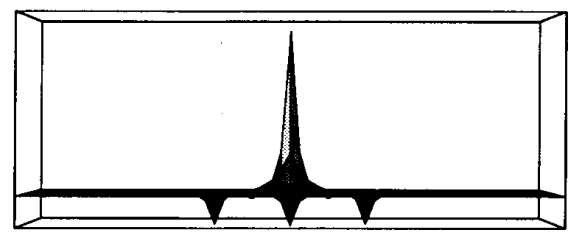

(b)

Fig. 13. (a) Sixth iteration of the filter in (83). (b) The same as (a) with a different viewpoint.

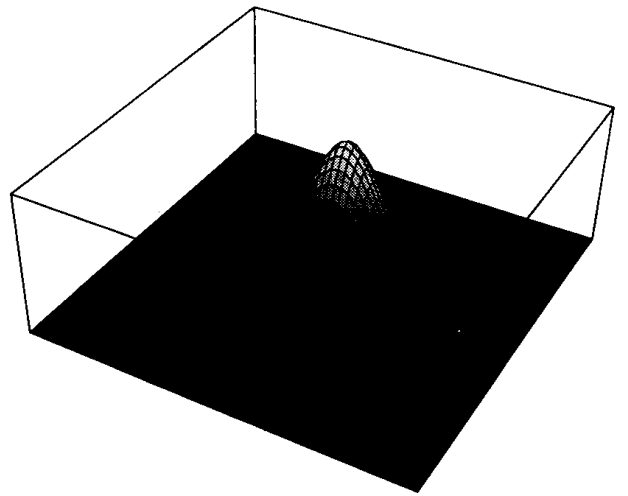

Fig. 14. Sixth iteration of a filter obtained when convolving (81) with itself. The plot is given on the rectangular grid.

ple dimensions still poses a number of challenges. Some initial results and conjectures on regularity were given, indicating the directions for future work.

\section{APPENDIX A}

\section{Notation AND Definitions}

This appendix establishes the notation used in the paper. Most of the definitions involving multidimensional $z$-transform and sampling are taken from [46].

1) Boldface lower/upper case letters will denote vectors and matrices, respectively. Raising an $n$-dimensional complex vector $z=\left(z_{1}, \cdots, z_{n}\right)$ to an $n$-dimensional integer vector $\boldsymbol{k}=\left(k_{1}, \cdots, k_{n}\right)$ yields

$$
z^{k}=z_{1}^{k_{1}} z_{2}^{k_{2}} \cdots z_{n}^{k_{n}}
$$


2) The $z$-transform of a discrete sequence $h(k)=$ $h\left(k_{1}, \cdots, k_{n}\right)$ is defined as

$$
H(z)=\sum_{k \in Z^{n}} h(k) z^{-k}
$$

while its Fourier transform is given by

$$
\hat{H}(\omega)=\sum_{\boldsymbol{k} \in \mathscr{P}^{n}} h(\boldsymbol{k}) e^{-j\langle\boldsymbol{\omega}, \boldsymbol{k}\rangle}
$$

where $\langle\boldsymbol{\omega}, \boldsymbol{k}\rangle$ denotes the inner product of the two vectors. Note that the Fourier transform of a sequence is its $z$-transform evaluated on the unit hypercircles.

3) Raising $z$ to a matrix power $\boldsymbol{D}$ denotes the following:

$$
z^{D}=\left(z^{d_{1}}, z^{d_{2}}, \cdots, z^{d_{n}}\right)
$$

where $d_{i}$ is the $i$ th column of the matrix $D$.

4) As an equivalent to the powers of the $N$ th root of unity, Viscito and Allebach in [46] define

$$
W_{D}(\omega)=\left(e^{-j\left\langle\omega, d_{1}\right\rangle}, \cdots, e^{-j\left\langle\omega, d_{n}\right\rangle}\right)
$$

with $d_{i}$ as given previously.

5) The Schur product of two vectors is given by the following:

$$
\boldsymbol{n} \circ \boldsymbol{k}=\left(n_{1} k_{1}, \cdots, n_{n} k_{n}\right) .
$$

6) $\tilde{H}(z)$ will mean transposition of the matrix, conjugation of coefficients and substitution of $z=\left(z_{1}, \cdots, z_{n}\right)$ by $\left(z_{1}^{-1} l, \cdots, z_{n}^{-1}\right)$, or equivalently substitution of $\boldsymbol{\omega}=$ $\left(\omega_{1}, \cdots, \omega_{n}\right)$ by $\left(-\omega_{1}, \cdots,-\omega_{n}\right)$, that is, complex conjugation on the unit circle. Note that we will assume real filter coefficients throughout.

7) Kronecker delta with vector subscripts $\delta_{n k}$ will be 1 only if the vector $(\boldsymbol{n}-\boldsymbol{k})$ is a zero vector.

8) FIR will stand for finite impulse response or compact support. The term orthogonal will be used in different contexts, among others to denote paraunitary (or lossless) matrices (see Section III-B). If we say that a filter is linear phase it will mean that the angle in its Fourier transform is a linear function of $\omega$, and this is possible if and only if the coefficients of its impulse response have central symmetry, i.e., if the corresponding polynomials are symmetric or antisymmetric.

9) If we say that polynomials $A_{i}$ satisfy the power complementary property it will mean

$$
\sum_{i} A_{i}(z) \tilde{A}_{i}(z)=1
$$

and the abbreviation PC will be used. In particular on the unit hypercircles $\left(z_{i}=e^{j \omega_{i}}, i=1, \cdots, n\right)$ (90) means that the magnitudes squared of the Fourier transforms of $A$ and $B$ sum up to one, i.e.,

$$
\sum_{i}\left|\hat{A}_{i}(\omega)\right|^{2}=1
$$

10) The identity matrix will be denoted by $I$, and the matrix with 1's along the antidiagonal by $J$.

\section{APPENDIX B}

Proof of Lemma 3.1

We prove the lemma point by point.

1) Noting that $p_{i}^{t}(z) x_{p}\left(z^{D}\right)=X(z)$ sufficiency is obvious since by substituting this condition into (12) one obtains $Y(z)=z^{-k_{N-1}} T(z) X(z)$ from where it can be seen that all the aliased versions of the input signal have disappeared. Here $\boldsymbol{k}_{N-1}$ denotes the vector from $\mathscr{U}_{c}^{t}$ which makes the vector of the inverse polyphase transform causal. To prove the necessity write $p_{i}^{t} \cdot T_{p}\left(z^{D}\right)=v^{t}(z)$. To cancel aliasing the output of the bank has to be of the form $Y(z)=$ $A(z) X(z)$ where $A(z)$ is just a scalar polynomial. Substituting $v(z)$ into (12) and equating it to $A(z) X(z)$

$$
\begin{aligned}
Y(z) & =z^{-k_{N-1}} p_{i}^{t}(z) \cdot T_{p}\left(z^{D}\right) \cdot x_{p}\left(z^{D}\right) \\
& =z^{-k_{N-t}} v^{t}(z) \cdot x_{p}\left(z^{D}\right)=A(z) \cdot X(z) .
\end{aligned}
$$

Substituting the expression for $X(z)$ as given above into the right-hand side of (92) yields

$$
\begin{aligned}
A(z) \cdot X(z) & =A(z) \cdot \sum_{k \in \mathscr{W}_{c}^{t}} z^{k} X_{k}\left(z^{D}\right) \\
& =\sum_{k \in \mathscr{H}_{c}^{t}} b_{k}(z) \cdot X_{k}\left(z^{D}\right), \\
& =b^{t}(z) \cdot x_{p}\left(z^{D}\right) \\
& =z^{-k_{N-1}} v^{t}(z) x_{p}\left(z^{D}\right)
\end{aligned}
$$

from where it is obvious that since $b_{k}(z)=A(z) \cdot z^{k}=$ $z^{-k_{N-1}} v_{k}(z)$ the vector $v(z)$ can be written as

$$
\begin{aligned}
v^{t}(z) & =\left(A(z) z^{k_{N-1}} z^{k_{0}} \cdots A(z) z^{k_{N-1}} z^{k_{N-1}}\right) \\
& =T(z) \cdot p_{i}^{t}
\end{aligned}
$$

which completes the proof of the first part.

2) If the system is perfect reconstruction then $Y(z)=$ $c z^{-n} X(z)$. When compared to $Y(z)=z^{-k_{N-1}} T(z) X(z)$ as obtained in the proof of the first part it is obvious that $T(z)=c \cdot z^{-n} z^{k_{N-1}}=c \cdot z^{-k}$. On the other hand, if $T(z)=c \cdot z^{-k}$ then substituting it into $Y(z)=$ $z^{-k_{N-1}} T(z) X(z)$ results in $Y(z)=z^{-k_{N-1}} \cdot c \cdot z^{-k}$. $X(z)=c \cdot z^{-n} \cdot X(z)$, i.e., perfect reconstruction is achieved.

3) Sufficiency is obvious since by choosing $G_{p}(z)=$ $\operatorname{Adj}\left(H_{p}(z)\right)$ the transfer matrix $T_{p}$ becomes a diagonal matrix of delays and

$$
\begin{aligned}
Y(z) & =z^{-k_{N-1}} p_{i}^{\prime}(z) \cdot z^{-k} \cdot I \cdot x_{p}\left(z^{D}\right) \\
& =z^{-n} \cdot X(z) .
\end{aligned}
$$

To prove the necessity, use the result of the second part of the lemma and write $T(z)=c \cdot z^{-k}$. Then it can be seen that $T_{p}\left(z^{D}\right)=c \cdot z^{-k} I$. Thus, $\operatorname{det} \boldsymbol{G}_{p} \operatorname{det} \boldsymbol{H}_{p}=\left(c \cdot \boldsymbol{z}^{-\boldsymbol{k}}\right)^{n}$ and since filters in both banks are FIR all the polynomial factors in both det $G_{p}$ and det $H_{p}$ have to be monomials.

\section{APPENDIX C}

Proof of Theorem 4.1 AND its Corollaries

This result for a one-dimensional system appeared in filter bank literature in [36] and in wavelet literature in [9], for a two-dimen- 
sional one in [43] and since the approach here is quite similar, we just outline the proof, for more details refer to [19].

Let us first prove a fact that will be used in the proof of the main theorem. Expanding the product in (17) yields

$$
\begin{aligned}
& \tilde{H}_{00}(z) H_{00}(z)+\tilde{H}_{10}(z) H_{10}(z)=1, \\
& \tilde{H}_{00}(z) H_{01}(z)+\tilde{H}_{10}(z) H_{11}(z)=0, \\
& \tilde{H}_{01}(z) H_{00}(z)+\tilde{H}_{11}(z) H_{10}(z)=0, \\
& \tilde{H}_{01}(z) H_{01}(z)+\tilde{H}_{11}(z) H_{11}(z)=1 .
\end{aligned}
$$

Proposition C.1: If the polyphase matrix is orthogonal, then $H_{00}$ and $H_{10}$ are relatively prime. Similarly, $H_{01}$ and $H_{11}$ are relatively prime.

Proof: Take the first statement. The second one is proved similarly. Suppose that $H_{00}$ and $H_{10}$ are not coprime. Then using the fact that a polynomial in $n$ variables which is not identically zero can be resolved into the product of irreducible factors in only one way [4], one can take the common factors out and call their product $P\left(z_{1}, z_{2}, \cdots, z_{n}\right)$. Substituting this into (96) results in

$$
P(z) \tilde{P}(z) \cdot\left(H_{00}^{\prime}(z) \tilde{H}_{00}^{\prime}(z)+H_{10}^{\prime}(z) \tilde{H}_{10}^{\prime}(z)\right)=1
$$

which for all the vanishing points of $P(z)$ goes to zero, contradicting the fact that the right side of (100) identically equals 1 .

Proof of Theorem 4.1: Consider (97). Since by Proposition C. $1 H_{00}$ and $H_{10}$ are relatively prime, one can conclude that $H_{11}(z)$ must contain all the polynomial factors of $\tilde{H}_{00}(z)$ except for the monomial $z^{k}$ which makes it causal. Thus, $H_{11}(z)=c_{1} \cdot z^{k}$. $\tilde{H}_{00}(z)$. Similarly $H_{10}(z)=c_{2} \cdot z^{k^{\prime}} \cdot \tilde{H}_{01}(z)$. Substituting this into (98) and cancelling $H_{00}(z) \tilde{H}_{01}(z)$ (it is allowed to do so since they are not identically equal to zero) yields $c_{2}=-\left(1 / c_{1}\right)$ and $\boldsymbol{k}^{\prime}=\boldsymbol{k}$. Thus, $H_{11}(z)=c \cdot z^{k} \cdot \tilde{H}_{00}(z)$, and $H_{10}(z)=-(1 / c) \cdot z^{k}$. $\tilde{H}_{01}(z)$. Substituting this into (99) and using (96) it can be seen that $c$ has to be either 1 or -1 . This finally gives us the complete specification of the system as given in the statement of the theorem.

Proof of Corollary 4.1: Take the polyphase components of the first filter (the proof for the second one is analogous). Since they are causal their corner coefficients are $P^{(0)}=P^{(1)}=(0, \cdots, 0)$ and $Q^{(0)}, Q^{(1)}$. Consider now the PC condition as given in the statement of the theorem. The polynomial $A(z)=H_{00}(z) \tilde{H}_{00}(z)$ will have the corner coefficients $P^{(A)}=-Q^{(0)}$ and $Q^{(A)}=Q^{(0)}$, while the corner coefficients of $B(z)=H_{01}(z) \tilde{H}_{01}(z)$ will be $P^{(B)}=-Q^{(1)}$ and $Q^{(B)}=Q^{(1)}$. For the PC property to hold the polynomials $A(z)$ and $B(z)$ must be of the same size for all coefficients (except the center one) to cancel, and thus both polyphase components have to be of the same size.

Proof of Corollary 4.2: Using the matrix $D_{1}$ from (38) write

$$
\tilde{H}_{0}(-z)=\tilde{H}_{00}\left(z^{D_{1}}\right)-z_{1} \tilde{H}_{01}\left(z^{D_{1}}\right)
$$

where $z^{D_{1}}=\left(z_{1} z_{2}, z_{2} z_{3}, \cdots, z_{1} z_{n}^{(-1)^{n-1}}\right)$. Using the expressions obtained in the proof of the theorem for the polyphase components of the second filter write

$$
\begin{aligned}
H_{1}(z)= & H_{10}\left(z^{D_{1}}\right)+z_{1}^{-1} H_{11}\left(z^{D_{1}}\right) \\
= & z_{1}^{-\left(k_{1}+k_{n}\right)} z_{2}^{-\left(k_{1}+k_{2}\right)} \cdots z_{n}^{-\left(k_{n-1}+(-1)^{n-1} k_{n}\right)} \\
& \cdot \tilde{H}_{01}\left(z^{D_{1}}\right) \\
& -z_{1}^{-1} z_{1}^{-\left(k_{1}+k_{n}\right)} z_{2}^{-\left(k_{1}+k_{2}\right)} \cdots z_{n}^{-\left(k_{n-1}+(-1)^{n-1} k_{n}\right)} \\
& \cdot \tilde{H}_{00}\left(z^{D_{1}}\right)
\end{aligned}
$$

$$
=-\boldsymbol{z}^{-\boldsymbol{k}^{\prime}} \tilde{H}_{0}(-\boldsymbol{z})
$$

\section{APPENDIX D}

\section{Proof of Lemma 5.2}

To prove the lemma a polynomial having a specific structure that will henceforth be denoted as an $A$-polynomial is first defined. A polynomial is an $A$-polynomial if it satisfies the following recursion:

$$
\begin{aligned}
A P^{(k)}\left(z_{n}\right)=A P^{(k)} & \left(z_{n-1}\right) \\
& +\sum_{i=1}^{k} A P^{(k-i)}\left(z_{n-1}\right) z_{1}^{-i}\left(z_{n}^{-i}+z_{n}^{i}\right)
\end{aligned}
$$

where

$$
A P^{(k)}\left(z_{1}\right)=z_{1}^{-2 k} A P^{(k)}\left(z_{1}^{-1}\right)=\sum_{i=0}^{k} p_{i} z_{1}^{-2 i}
$$

Here $z_{i}=\left(z_{1}, \cdots, z_{i}\right)$. Note that in one dimension an $A$-polynomial is just a symmetric polynomial having only even degree terms, in two dimensions it is a diamond shaped symmetric polynomial having again just even degree terms, a.s.o. Also note that the sum of two $A$-polynomials of the same degree is again an $A$-polynomial. $A$ prefix " $A$ " associated with the name of the polynomial will indicate that it is an $A$-polynomial, a superscript indicates its degree (superscript (0) would mean a constant). By $d_{c}$ the degree of the center coefficient will be denoted (the degree is the sum of all the exponents) and by $a_{c}$ the coefficient itself. Here the statements of some facts that will be used in the proof of the lemma are given. Since their proofs are just technical they are omitted here (for details, see [18]).

Proposition D.1: For $A P^{(k)}$ the following is true:

1) it is of size $(2 k+1)$ in all dimensions;

2) it has just even degree terms;

3) $i\left(d_{c}\right)=i(k)$;

4) $A P^{(k)}\left(z_{n}\right)=z_{1}^{-2 k} A P^{(k)}\left(z_{n}^{-1}\right)$, i.e., all $A$-polynomials are symmetric polynomials;

5) $z_{1}^{-2 m} A P^{(k)}=A Q^{(k+2 m)}$;

6) $A P^{(k)} A Q^{(m)}=A R^{(k+m)}$.

For the sake of clarity in the statement of the lemma the prefix " $A$ " was omitted as well as the superscripts associated with the polynomials. For the proof they are reintroduced. Also note that the whole analysis will be performed in the upsampled domain.

Proof:

1) To prove the lemma let us write the general expression for the polyphase matrix containing polyphase components of sizes 3 and 5 in $n$ dimensions (recall that $z_{u}^{(i)}$ denotes $z^{D}$ where $z=$ $\left.\left(z_{1}, \cdots, z_{i}\right)^{t}\right)$

$$
H_{p}\left(z_{u}^{(n)}\right)=\left(\begin{array}{ll}
A H_{00}^{(1)}\left(z_{u}^{(n)}\right) & A H_{01}^{(0)}\left(z_{u}^{(n)}\right) \\
A H_{10}^{(2)}\left(z_{u}^{(n)}\right) & A H_{11}^{(1)}\left(z_{u}^{(n)}\right)
\end{array}\right)
$$

Due to their required size and the fact that they have to be linear phase it is obvious that the above polyphase components indeed are $A$-polynomials. Therefore, we can expand each one using (101). $A H_{01}^{(0)}\left(z_{u}^{(n)}\right)$ having a superscript (0) is obviously a constant that will henceforth be denoted by $b$. Note that (101) implies that the first term can be taken as the corresponding polyphase component in $(n-1)$ dimensions. Then the determinant of (103) can be written 


$$
\begin{aligned}
\operatorname{det} \boldsymbol{H}_{p}\left(z_{u}^{(n)}\right)= & A H_{00}^{(1)}\left(z_{u}^{(n)}\right) A H_{11}^{(1)}\left(z_{u}^{(n)}\right)-A H_{10}^{(2)}\left(z_{u}^{(n)}\right) b \\
= & \underbrace{\frac{\operatorname{det} H_{p}\left(z_{u}^{(n-1)}\right)}{A H_{00}^{(1)}\left(z_{u}^{(n-1)}\right) A H_{11}^{(1)}\left(z_{u}^{(n-1)}\right)-A H_{10}^{(2)}\left(z_{u}^{(n-1)}\right) b}+2 a d z_{1}^{-2}}_{T_{1}} \\
& +\underbrace{\left(a \cdot A H_{11}^{(1)}\left(z_{u}^{(n-1)}\right)+d \cdot A H_{00}^{(1)}\left(z_{u}^{(n-1)}\right)-b \cdot A H_{c}^{(1)}\left(z_{u}^{(n-1)}\right)\right)}_{T_{2}} \cdot z_{1}^{-1}\left(z_{n}^{-1}+z_{n}\right) \\
& +\underbrace{(a d-b c)}_{T_{3}} \cdot z_{1}^{-2}\left(z_{n}^{-2}+z_{n}^{2}\right) \cdot
\end{aligned}
$$

a) Let us first consider the case when $b \neq 0$. Observe that $T_{2}$ and $T_{3}$ have to equal zero since they appear in pairs, and thus

$$
\begin{gathered}
a \cdot d=b \cdot c \\
A H_{c}^{(1)}\left(z_{u}^{(n-1)}\right) \\
=\frac{d \cdot A H_{00}^{(1)}\left(z_{u}^{(n-1)}\right)+a \cdot A H_{11}^{(1)}\left(z_{u}^{(n-1)}\right)}{b} .
\end{gathered}
$$

Here we still do not know what $b, A H_{00}^{(1)}\left(z_{u}^{(n-1)}\right)$, and $A H_{11}^{(1)}\left(z_{u}^{(n-1)}\right)$ are. Substituting (105) and (106) into (104) we are left with

$$
\operatorname{det} \boldsymbol{H}_{p}\left(\boldsymbol{z}_{u}^{(n)}\right)=\operatorname{det} \boldsymbol{H}_{p}\left(z_{u}^{(n-1)}\right)+2 a d z_{1}^{-2}
$$

which obviously has to have a single nonzero coefficient. Using Proposition D. 1 it can be shown that det $\boldsymbol{H}_{p}\left(\boldsymbol{z}_{u}^{(n-1)}\right)$ is again an $A$-polynomial with index (2) and it possesses a center term $z_{1}^{-2}$. Hence, we just have to find a solution which would make this $A$-polynomial become a single term. Note that achieving this is equivalent to finding a general solution in $(n-1)$ dimensions, which in turn yields $b, A H_{00}^{(1)}\left(z_{u}^{(n-1)}\right), A H_{11}^{(1)}\left(z_{u}^{(n-1)}\right)$ and finally $A H_{c}^{(1)}\left(z_{u}^{(n-1)}\right)$. This proves the lemma for the case when $b$ is nonzero.

b) We want to show now by contradiction that $b$ cannot be zero. Thus suppose that $b=0$. This means that $a d=0$ as well, i.e., $a=0$ or $d=0$ or both. Without loss of generality suppose that $a=0$. Plugging all this into $T_{2}$ we see that $d \cdot A H_{00}^{(1)}\left(z_{u}^{(n-1)}\right)$ has to equal zero as well since it appears in pairs. Now $A H_{00}^{(1)}\left(z_{u}^{(n-1)}\right)$ cannot be zero since otherwise the whole determinant would be zero as well. Thus, we conclude that both $a$ and $d$ have to be zero. What we want to show now is that the only term left, i.e., $A H_{00}^{(1)}\left(z_{u}^{(n-1)}\right) A H_{11}^{(1)}\left(z_{u}^{(n-1)}\right)$ cannot have a single nonzero coefficient. To do this we can express each of these $A$ polynomials in terms of $A$-polynomials in $(n-2)$ dimensions and upon multiplying them observe that forcing the coefficient that appear in pairs to be zero, we are left with $A H_{00}^{(1)}\left(z_{u}^{(n-2)}\right) A H_{11}^{(1)}\left(z_{u}^{(n-2)}\right)$. Continuing this procedure we finally arrive to the same product in one dimension for which it is trivial to show that it cannot be forced to have a single nonzero coefficient.

2) To prove the second part of the lemma we have to use double induction, on the number of dimensions and on $k$. Both in the first and in the inductive step on $n$, the first step for $k=1$ is trivial since it is just the $3 / 5$ solution from the first part of the lemma. Thus, in each case, we will consider only inductive step on $k$. Also bear in mind that each polyphase component in the $3 / 5$ solution from the first part of the lemma is an $A$-polynomial.

- $n=1$ :

$$
\begin{gathered}
\left(\begin{array}{cc}
A H_{00}^{(k-1)}\left(z_{1}^{2}\right) & A H_{01}^{(k-2)}\left(z_{1}^{2}\right) \\
A H_{10}^{(k)}\left(z_{1}^{2}\right) & A H_{11}^{(k-1)}\left(z_{1}^{2}\right)
\end{array}\right) \\
\cdot\left(\begin{array}{cc}
A H_{00}^{(1)}\left(z_{1}^{2}\right) & A H_{01}^{(0)}\left(z_{1}^{2}\right) \\
A H_{10}^{(2)}\left(z_{1}^{2}\right) & A H_{11}^{(1)}\left(z_{1}^{2}\right)
\end{array}\right) \\
=\left(\begin{array}{cc}
A H_{00}^{(k)}\left(z_{1}^{2}\right) & A H_{01}^{(k-1)}\left(z_{1}^{2}\right) \\
A H_{10}^{(k+1)}\left(z_{1}^{2}\right) & A H_{11}^{(k)}\left(z_{1}^{2}\right)
\end{array}\right)
\end{gathered}
$$

where we used the results on $A$-polynomials stated previously.

- Inductive step: The proof is completely analogous to the first step.

\section{ACKNOWLEDGMENT}

The authors would like to thank Dr. I. Daubechies and Dr. A. Cohen of AT\&T Bell Labs for useful discussions.

\section{REFERENCES}

[1] R. Ansari, "Two-dimensional IIR filters for exact reconstruction in tree-structured subband decomposition," Electron. Lett., vol. 23, no. 12 , pp. 633-634, June 1987.

[2] R. Ansari, H. Gaggioni, and D. J. LeGall, "HDTV coding using a non-rectangular subband decomposition," in Proc. SPIE Conf. Vis. Commun. Image Processing, Cambridge, MA, Nov. 1988, pp. $821-824$.

[3] M. Antonini, M. Barlaud, and P. Mathieu," Image coding using lattice vector quantization of wavelet coefficients, " in Proc. IEEE Int. Conf. ASSP, Toronto, Canada, May 1991, pp. 2273-2276.

[4] M. Bocher, Introduction to Higher Algebra. New York Macmillan, 1907.

[5] J. W. Cassels, An Introduction to the Geometry of Numbers. Berlin; Springer-Verlag, 1971.

[6] T. Chen and P. P. Vaidyanathan, "Multidimensional multirate filters derived from one-dimensional filters," Electron. Lett., Jan. 1991.

[7] A. Cohen and I. Daubechies, "Nonseparable bidimensional wavelet bases," submitted to Rev. Math. Iberoamericana, 1991.

[8] A. Croisier, D. Esteban, and C. Galand, "Perfect channel splitting by use of interpolation/decimation/tree decomposition techniques," in Int. Conf. Inform. Sci. Syst., Patras, Greece, Aug. 1976, pp. $443-446$.

[9] I. Daubechies, "Orthonormal bases of compactly supported wavelets," Commun. Pure Appl. Math., vol. 41, pp. 909-996, Nov, 1988.

[10] I. Daubechies and A. Cohen, Private communication.

[11] I. Daubechies and J. Lagarias, "Two-scale difference equations: II. Local regularity, infinite products of matrices and fractals," SIAM J. Math. Anal., 1992, to appear.

[12] G. Deslauriers, J. Dubois, and S. Dubuc, "Multidimensional iterative interpolation," Can. J. Math., vol. 43, no. 2, pp. 297-312, 1991. 
[13] E. Dubois, "The sampling and reconstruction of time-varying imagery with application in video systems," Proc. IEEE, vol. 73, no. 4, pp. 502-522, Apr. 1985.

[14] D. E. Dudgeon and R. M. Mersereau, Multidimensional Digital Signal Processing. Englewood Cliffs, NJ; Prentice-Hall 1984.

[15] J.-C. Feauveau, "Analyse multirésolution par ondelettes non orthogonales et bancs de filtres numériques,'” Ph.D. dissertation, Univ. Paris Sud, 1990.

[16] K. Gröchenig and W. R. Madych, "Multiresolution analysis, Haar bases and self-similar tilings of $R^{n}$," IEEE Trans. Inform. Theory, vol. 38, pt. II, pp. 556-568, Mar. 1992.

[17] A. Grossmann and J. Morlet, "Decomposition of Hardy functions into square integrable wavelets of constant shape," SIAM J. Math. Anal., vol. 15, no. 4, pp. 723-736, 1984.

[18] J. Kovačević, "Filter Banks and Wavelets: Extensions and Applications," Ph.D. dissertation, Columbia Univ., New York, 1991.

[19] J. Kovačević and M. Vetterli, "A theory of multidimensional multirate FIR filter banks," Center for Telecommun. Res. Elect. Eng. Dep., Columbia Univ., Tech. Rep., 1991.

[20] J. Kovačević, M. Vetterli, and G. Karlsson, "Design of multidimensional filter banks for non-separable subsampling," in Proc. IEEE Int. Symp. Circuits Syst., New Orleans, LA, May 1990, pp. 2004-2008.

[21] G. Karlsson and M. Vetterli, "Theory of two-dimensional multirate filter banks," IEEE Trans. Acoust., Speech, Signal Processing, vol. 38, no. 6, pp. 925-937, June 1990.

[22] G. Karlsson, M. Vetterli, and J. Kovačević, "Non-separable twodimensional perfect reconstruction filter banks," in Proc. SPIE Conf. Vis. Commun. Image Processing, Cambridge, MA, Nov. 1988 , pp. $187-199$.

[23] S. Mallat, "A theory of multiresolution signal decomposition: The wavelet representation," IEEE Trans. Pattern Recognition Machine Intell., vol. 11, no. 7, pp. 674-693, July 1989.

[24] J. McClellan, "The design of two-dimensional filters by transformations, in Proc. 7th Ann. Princeton Conf. ISS, Princeton, NJ, 1973 pp. 274-251.

[25] Y. Meyer, Ondelettes. Paris; Hermann, 1990.

[26] F. Mintzer, "Filters for distortion-free two-band multirate filter banks," IEEE Trans. Acoust., Speech, Signal Processing, vol. ASSP-33, no. 3, pp. 626-630, June 1985 .

[27] M. Newman, Integral Matrices. New York: Academic, 1972.

[28] T. Q. Nguyen and P. P. Vaidyanathan, "Two-channel perfectreconstruction FIR QMF structures which yield linear-phase analysis and synthesis filters," IEEE Trans. Acoust., Speech, Signal Processing, vol. 37, no. 5, pp. 676-690, May 1989.

[29] O. Rioul, "Dyadic up-scaling schemes: Simple criteria for regularity," SIAM J. Math. Anal., Feb. 1991, submitted for publication.

[30] M. J. T. Smith and T. P. Barnwell III, "Exact reconstruction for tree-structured subband coders," IEEE Trans. Acoust., Speech, Signal Processing, vol. ASSP-34, no. 3, pp. 431-441, June 1986.

[31] - , "A new filter bank theory for time-frequency representation,", IEEE Trans. Acoust., Speech, Signal Processing, vol. ASSP-35, no. 3, pp. 314-327, Mar. 1987.
[32] G. Strang, "Wavelets and dilation equations: A brief introduction," SIAM Rev., vol. 31, no. 4, pp. 614-627, Dec. 1990.

[33] P. P. Vaidyanathan, "Theory and design of M-channel maximally decimated quadrature mirror filters with arbitrary $M$, having the perfect reconstruction property," IEEE Trans. Acoust., Speech, Signal Processing, vol. ASSP-35, no. 4, pp. 476-492, Apr. 1987.

[34] - Robust Digital Filters and Multirate Filter Banks. Englewood Cliffs, NJ: Prentice Hall, 1992, to appear.

[35] P. P. Vaidyanathan and P. Q. Hoang, "Lattice structures for optimal design and robust implementation of two-channel perfect reconstruction filter banks," IEEE Trans. Acoust., Speech, Signal Processing, vol. 36, no. 1, pp. 81-94, 1988.

[36] P. P. Vaidyanathan and Z. Doğanata, "The role of lossless systems in modern digital signal processing: A tutorial," IEEE Trans. Education, vol. 32, no. 3, pp. 181-197, Aug. 1989

[37] M. Vetterli, "Multi-dimensional sub-band coding: Some theory and algorithms," Signal Processing, vol. 6, no. 2, pp. 97-112, Feb. 1984.

[38] - , "Filter banks allowing perfect reconstruction," Signal Processing, vol. 10, no. 3, pp. 219-244, Apr. 1986.

[39] - "A theory of multirate filter banks," IEEE Trans. Acoust., Speech, Signal Processing, vol. ASSP-35, pp. 356-372, Mar. 1987.

[40] -, "Multirate filter banks for subband coding," in Subband Image Coding, J. W. Woods, Ed. New York: Kluwer, 1990.

[41] - "Wavelets and filter banks for discrete-time signal processing," in Wavelets and Their Applications, R. Coifman et al., Eds. New York: Jones and Bartlett, 1991.

[42] M. Vetterli and C. Herley, "Wavelets and filter banks: Theory and design," IEEE Trans. Acoust., Speech, Signal Processing, to appear Sept. 1992

[43] M. Vetterli, J. Kovačević, and D. J. LeGall, “Perfect reconstruction filter banks for HDTV representation and coding," Image Commun., vol. 2 , no. 3, pp. $349-364$, Oct. 1990

[44] M. Vetterli and D. J. LeGall, "Perfect reconstruction FIR filter banks: some properties and factorizations," IEEE Trans. Acoust., Speech, Signal Processing, vol. 37, no. 7, pp. 1057-1071, July 1989.

[45] E. Viscito and J. P. Allebach, "Design of perfect reconstruction multi-dimensional filter banks using cascaded Smith form matrices," in Proc. IEEE Int. Symp. Circuits Syst., Espoo, Finland, June 1988, pp. 381-384

[46] E. Viscito and J. P. Allebach, "The analysis and design of multidimensional FIR perfect reconstruction filter banks for arbitrary sampling lattices," IEEE Trans. Circuits Syst., vol, 38, no. 1, pp. 29-42, Jan. 1991

[47] J. W. Woods and S. D. O'Neil, "Sub-band coding of images," IEEE Trans. Acoust., Speech, Signal Processing, vol. ASSP-34, pp. 1278-1288, Oct. 1986.

[48] E. P. Simoncelli and E. H. Adelson, "Non-separable extensions of quadrature mirror filters to multiple dimensions,' Proc. IEEE, vol. 78, pp. 652-664, Apr. 1990.

[49] W. M. Lawton and H. L. Resnikoff, "Multidimensional wavelet bases," submitted to SIAM J. Anal., 1991. 